\title{
Characteristics of the electrojet during intense magnetic disturbances
}

\author{
Liudmila I. Gromova ${ }^{1}$, Matthias Förster ${ }^{2,3}$, Yakov I. Feldstein ${ }^{1}$, and Patricia Ritter ${ }^{2}$ \\ ${ }^{1}$ Institute of Terrestrial Magnetism, Ionosphere, and Radiowave Propagation of the Russian Academy of Sciences \\ (IZMIRAN), 142090 Troitsk, Moscow region, Russia \\ ${ }^{2}$ Helmholtz Centre Potsdam, GFZ German Research Centre for Geosciences, 14473 Potsdam, Germany \\ ${ }^{3}$ Max Planck Institute for Solar System Research, 37077 Göttingen, Germany
}

Correspondence: Matthias Förster (mfo@gfz-potsdam.de)

Received: 6 April 2018 - Discussion started: 25 April 2018

Revised: 30 September 2018 - Accepted: 4 October 2018 - Published: 17 October 2018

\begin{abstract}
Hall current variations in different time sectors during six magnetic storms from the summer seasons in 2003 and 2005 (Ritter, 2018) are examined, namely three storms in the day-night meridional sector and three storms in the dawn-dusk sector. The sequence of the phenomena, their structure and positions, and the strength of the polar (PE) and the auroral (AE) Hall electrojets were investigated using scalar magnetic field measurements obtained from the CHAllenging Minisatellite Payload (CHAMP) satellite in accordance with the study of Ritter et al. (2004a). We analyzed the correlations of the PE and AE as well as the obtained regression relations of the magnetic latitude MLat and the electrojet current intensity $I$ with auroral and ring current activity, the interplanetary magnetic field, and the Newell et al. (2007) coupling function for the state of the solar wind. The following typical characteristics of the electrojets were revealed:
\end{abstract}

The PE appears in the daytime sector at MLat $\sim 80^{\circ}-$ $73^{\circ}$, with a westward or an eastward direction depending on the interplanetary magnetic field (IMF) $B_{y}$ component $\left(B_{y}<0 \mathrm{nT}\right.$ or $\left.B_{y}>0 \mathrm{nT}\right)$. Changes in the current flow direction in the $\mathrm{PE}$ can occur repeatedly during the storm, but only due to changes in the IMF $B_{y}$ orientation. The PE increases with the intensity of the IMF $B_{y}$ component from $I \sim 0.4 \mathrm{~A} \mathrm{~m}^{-1}$ for $B_{y} \sim 0 \mathrm{nT}$ up to $I \sim 1.0 \mathrm{~A} \mathrm{~m}^{-1}$ for $B_{y} \sim 23 \mathrm{nT}$. The MLat position of the PE does not depend on the direction and intensity of the $B_{y}$ component.

There is no connection between MLat and $I$ in the PE and the symmetric part of the magnetospheric ring current (index SymH). There is a correlation between $I$ in the PE and the
AsyH index, but only a very weak interconnection of this index with the MLat of the PE.

Substorms occurring before the storm's main phase are accompanied by the appearance of an eastward electrojet (EE) at MLat $\sim 64^{\circ}$ as well as that of a westward electrojet (WE). In the nighttime sector, a WE appears at MLat $\sim 64^{\circ}$. During the main phase both electrojets persist. The daytime EE and the nighttime WE shift toward sub-auroral latitudes of MLat $\sim 56^{\circ}$ and grow in intensity up to $I \sim 1.5 \mathrm{~A} \mathrm{~m}^{-1}$. The $\mathrm{WE}$ is then located about $6^{\circ}$ closer to the pole than the $\mathrm{EE}$ during evening hours and about $2^{\circ}-3^{\circ}$ closer during daytime hours.

\section{Introduction}

The pioneering work of Dungey (1961) about the open nature of the magnetosphere and the role of magnetic reconnection processes between the interplanetary magnetic field (IMF) and the geomagnetic main field paved the way for the understanding of the large-scale structure and dynamics of the Earth's spatial environment. It provided the theoretical framework for subsequent successful investigations to understand the solar-wind-magnetosphere-ionospherethermosphere coupling processes over several decades (Cowley, 2015).

The reconnection processes on the dayside give rise to open magnetic flux areas, the polar caps, and together with the nightside reconnections, they are driving a large-scale internal magnetospheric plasma convection and current sys- 
tems that connect magnetospheric and ionospheric domains. Due to the large variability of the solar wind and IMF conditions, the whole system is very dynamic and the amount of open flux in the polar cap changes continuously. The theoretical understanding of the polar cap formation and its time evolution was advanced to the fully time-dependent "expanding-contracting polar cap" (ECPC) paradigm by the work of Cowley and Lockwood (1992). A comprehensive review of all the aspects of the magnetosphere-ionospherethermosphere interaction processes under the solar wind driver was provided recently by Milan (2015).

The principal pattern of the large-scale field-aligned current (FAC) system, also known as Birkeland currents, which form the circumpolar belts of Region-1 (on the poleward side) and Region-2 FACs (on the equatorward side), was disclosed by the work of Iijima and Potemra (1976a). On the dayside adjacent to the cusp region, so-called Region-0 FACs are observed (Iijima and Potemra, 1976b). Using MAGSAT satellite data, Iijima et al. (1984) showed the existence of a particular FAC system in the dayside sector of the polar cap, the so-called NBZ Birkeland currents for intervals of positive IMF $B_{z}$.

From ground-based magnetometer observations, the highlatitude electrojets have already been studied prior to the space era (cf. Chapman and Bartels, 1940). The particularities of high-latitude ionospheric current systems during magnetic disturbances were known empirically (Chapman, 1935). Levitin et al. (1982) revealed high-latitude current systems for summer conditions, which are controlled by the IMF components and solar wind parameters. Based on such equivalent current systems and considering information about the anisotropic ionospheric conductivity, FriisChristensen et al. (1985) showed the possibility to determine a full current system, including FACs and horizontal currents in the ionosphere. Such a full current system can be estimated as a combination of the equivalent currents obtained both from ground-based and satellite observations of the magnetic variations (Green et al., 2007).

An intense study of the polar electrojet (PE) at the highlatitude daytime ionosphere was initiated by the works of Svalgaard (1968) and Mansurov (1969). They demonstrated that its characteristic magnetic field variation depends on the sector structure of the IMF that is much like the average magnetic field of the solar photosphere. The IMF sector structure does not always correspond to the expected magnetic field variations in the near-polar region. Friis-Christensen et al. (1972) showed that during periods of discrepancy between the expected magnetic variations and the sector structure from satellite observations, an essential deviation of the IMF from the usual spiral structure always existed. During these cases, the azimuthal IMF $B_{y}$ component was oppositely directed to the expected direction of the spiral. This implies that the magnetic variation on the ground is not primarily controlled by the sector structure (toward or away the sun), but by the azimuthal component of the IMF (eastward or westward).

Various methods have been developed for the extraction of the PE magnetic field variations from ground-based observations in the near-polar region (Feldstein, 1976). The most effective approach appeared to be the correlation method (Jørgensen et al., 1972; Friis-Christensen and Wilhjelm, 1975; Feldstein et al., 1975b). It is based on the fact that both the direction of the PE and its intensity depend on the IMF $B_{y}$ component. The method allows for the separation of the magnetic variations of the PE from variations of other sources and shows the spatial-temporal variation of the PE vector variations very clearly. Feldstein et al. (1975b) described the findings of a geomagnetically quiet interval in summer 1965 and the characteristics of the equivalent current system controlled by the IMF $B_{y}$ component. In a first step, time intervals with correlations of the magnetic $X(H), Y$, and $Z$ components with IMF $B_{y}$ were identified for observatories with $\Phi>65^{\circ}$. In case of existing correlations, they always appeared to be practically close to a linear dependence with a correlation coefficient $r$. Correlation was assumed to exist for values of $r>0.4$; otherwise (for $r \leq 0.4$ ) it was assumed as non-existing. Such a boundary for significant $r$ values is justified by the correlation correction $S_{r}=\left(1-r^{2}\right) / \sqrt{(n-1)}$. For values of $\left|r / S_{r}\right| \geq 3$, the relation between the $X(H), Y$, and $Z$ components with the IMF $B_{y}$ cannot be regarded as accidental. With $n \sim 50$ the correlation is not randomly distributed for $r>0.4$ (corresponding to $95 \%$ confidence interval).

Regression lines, which relate the ground magnetic variations with the IMF $B_{y}$ component, were estimated for all MLTs, based on the observed intervals with $r>0.4$. They were used to describe the spatial-temporal distribution of the surface magnetic variations in the horizontal and vertical plane, and were finally used for the estimation of the equivalent current system for IMF $B_{y}=6 \mathrm{nT}$. Its integral intensity amounts to $180 \mathrm{kA}$, with a maximum current intensity of the electrojet in the dayside sector of $\sim 0.5 \mathrm{~A} \mathrm{~m}^{-1}$ at $80^{\circ}<\Phi<81^{\circ}$. An analogous estimation for July-August 1966 resulted in a value of $\sim 0.35 \mathrm{~A} \mathrm{~m}^{-1}$ at the same latitudes (Sumaruk and Feldstein, 1973).

The PE in the dayside sector does not disappear during magnetic disturbances (Feldstein et al., 2006). The PE shifts equatorward to $72^{\circ}<\Phi<74^{\circ}$ in the longitudinal range of 08:00 $<$ MLT $<17: 00$ during intense substorms (with AL $800 \mathrm{nT}$ ), and during periods of geomagnetic storms with $\mathrm{AL} \sim-1200 \mathrm{nT}$ and Dst $\sim-150 \mathrm{nT}$, it is situated at $66^{\circ}<$ $\Phi<68^{\circ}$ between 09:00 $<$ MLT $<15: 00$. The current intensities of the PE increase only slightly to about $\sim 0.5 \mathrm{~A} \mathrm{~m}^{-1}$.

The variations of the magnetic field at the Earth's surface at high latitudes, which were derived with the method of regression analysis, allowed for the determination of the IMF $B_{y}$ control of the spatial-temporal distributions of the electric field potential at ionospheric altitudes as well as the ionospheric and field-aligned currents (FACs; (Friis-Christensen 
et al., 1985; Feldstein and Levitin, 1986)). The electric field potential for an inhomogeneous ionospheric conductivity is obtained by solving a second-order partial differential equation. Friis-Christensen et al. (1985) used magnetic observations of the summer seasons in 1972 and 1973, while Feldstein and Levitin (1986) obtained it for summer 1968. The potential differences at cusp latitudes in the daytime sector are $\sim 20 \mathrm{kV}$ for IMF $B_{y} \sim \pm 6 \mathrm{nT}$.

Olsen (1996) used MAGSAT magnetic field data in a height range of $350<\mathrm{h}<550 \mathrm{~km}$ to determine the strength and location of the auroral electrojets at $115 \mathrm{~km}$ altitude. For the first time, he showed the possibility of estimating the horizontal ionospheric currents from scalar magnetic measurements only. The ionospheric currents were modelled by hundreds of infinite linear currents perpendicular to the orbital plane of the spacecraft, with discretization intervals of $111 \mathrm{~km}$. The problem of ionospheric current estimation is underdetermined, and its solution is not unique. In order to constrain the solution, a regularization method is used. The comparison of modelled and measured variations of the magnetic field along the satellite orbit on 04 December 1979 at 17:00 UT demonstrates the good agreement for the field-aligned component but a significant discrepancy for the field-perpendicular one. The discrepancy is mainly caused by magnetic fields of the FACs. The integral amplitude of the ionospheric currents during the interval from 28 November to 10 December 1979 yielded a correlation of $r=0.88$ with the AE-index.

The IMF $B_{y}$ orientation influences not only the PE but also the movements of the auroral forms at cusp latitudes (Sandholt et al., 2002). Simultaneous with permanently polewardmoving discrete auroral forms at the equatorward boundary of the cusp, which are controlled by the IMF $B_{z}$ component, east-west moving auroral forms exist. This azimuthal movement is controlled by IMF $B_{y}$, such that for $B_{y}>0$, the discrete forms move westward, and for $B_{y}<0$ they move eastward. The movement of the auroral forms is in an opposite direction to the PE current flow direction. This can be expected, because the discrete auroral forms and the channels of enhanced ionospheric conductivity are both due to precipitating electrons into the upper atmosphere. A detailed consideration of the interrelation between auroral luminosity, auroral particle precipitation, and the PE during magnetic disturbances was given by Sandholt et al. (2004). As shown there, the strong convection channel is located on the dawn side of the polar cap for IMF $B_{y}>0$ and on the dusk side for $B_{y}<0$ conditions. The electron precipitation in the regime of the convection channel in the morning sector consists of a band $(\sim 500 \mathrm{~km})$ of structured precipitation. The PE is located on the high-latitude boundary of the structured luminosity region in the vicinity of the strong flow channel of magnetospheric convection close to the bright auroral arc. For $B_{y}>0$, this channel is located in the morning sector on the poleward side of the polar cap boundary, with FAC out of the ionosphere and FAC into the ionosphere equatorward of the polar cap boundary.

Ritter et al. (2004b) investigated variations in the location and density of the auroral electrojets, which were independently determined from both the ground-based (IMAGE magnetometer network) and satellite (CHAllenging Minisatellite Payload - CHAMP) measurements.

Wang et al. (2008) made use of the Hall current estimations for the intense magnetic storms on 31 March to 01 April 2001 and 17 April-21 April 2002 to investigate the position and current densities of auroral electrojets (westward electrojet, WE, and eastward electrojet, EE) as well as the relations of the electrojets to the Dst index and the IMF $B_{z}$ component. The characteristics of the PE have not been considered by these authors. The currents were determined from scalar magnetic field measurements of the CHAMP satellite (orbit in the meridional plane of 15:00-03:00 and 16:0004:00 MLT) according to the method of Ritter et al. (2004b). The intensity of the WE on the nightside is, on average, 2 times larger than the EE on the dayside.

In this study we investigate not only the auroral electrojet, but also the polar electrojet characteristics during six intense magnetic summer storms. In Sect. 2 we present an overview of the CHAMP data used as well as the indices, which characterize the electromagnetic conditions in the near-Earth space during the geomagnetic storms under study. Section 3 provides a short description of the method for the determination of the Hall currents from CHAMP scalar magnetic records. In Sect. 4 we consider the latitudinal variation of the strength and position of the electrojets during different phases of the magnetic storm on 29 May 2003-30 May 2003. Particular attention is drawn to the polar electrojet (PE). The subsequent Sect. 5 provides detailed correlation analyzes and the discussion of the control of the current direction in the electrojets, its strength, and its latitudinal position by various indices. The Conclusion's Sect. 6 summarizes the main results of the study with respect to the Hall current variations during the various storm phases.

\section{Data}

The CHAllenging Minisatellite Payload (CHAMP) spacecraft (Reigber et al., 2002) was launched on 15 July 2000 into a circular, near-polar orbit with an inclination of $87.3^{\circ}$. From its initial orbital height at $\sim 460 \mathrm{~km}$, it has decayed to $\sim 400 \mathrm{~km}$ in 2003 and $\sim 350 \mathrm{~km}$ after 5 years. The orbital plane precesses to earlier local times at a rate of about $1 \mathrm{~h}$ per 11 days, so that the orbit covers all local times within about 131 days. The data used in this study are scalar magnetic field measurements obtained with the Overhauser magnetometer (OVM) at the boom tip with a resolution of $0.1 \mathrm{nT}$. In order to isolate the magnetic effect of ionospheric currents in the satellite data, the contributions from all other sources have been removed from the scalar field readings, as described in the study of Ritter et al. (2004a). 
Table 1. Overview of CHAMP satellite orbits used for this study.

\begin{tabular}{lrrr}
\hline Date and Time (UT, hrs.) & $\begin{array}{r}\text { CHAMP } \\
\text { orbit numbers }\end{array}$ & $\begin{array}{r}\text { MLT range (hrs.) } \\
\text { ascending }\end{array}$ & descending \\
\hline 29/30 May 2003, 16:00-10:00 & $16229-16240$ & $\sim 14: 00-16: 00$ & $\sim 02: 00-04: 00$ \\
24 Aug 2005, 07:00-20:00 & $29012-29020$ & $\sim 11: 00-12: 00$ & $\sim 23: 00-24: 00$ \\
18 Jun 2003, 03:00-18:00 & $16532-16541$ & $\sim 12: 00-16: 00$ & $\sim 00: 00-04: 00$ \\
30 May 2005, 02:00-17:00 & $27658-27667$ & $\sim 19: 00-21: 00$ & $\sim 06: 00-09: 00$ \\
15 May 2005, 00:00-19:00 & $27423-27432$ & $\sim 20: 00-22: 00$ & $\sim 08: 00-10: 00$ \\
18 Aug 2003, 00:00-23:00 & $17480-17494$ & $\sim 07: 00-09: 00$ & $\sim 19: 00-21: 00$ \\
\hline
\end{tabular}

The CHAMP orbital intervals during various storm periods used for this study are listed in Table 1. The quantity, locations, and intensity of the peaks along the latitudinal current intensity distribution vary over the course of the storm development. For the description of the storm development, we utilize various solar and geomagnetic indices.

First, we employ the auroral electrojet index (AE), which is derived from geomagnetic variations in the horizontal component observed at 12 selected observatories along the auroral zone in the Northern Hemisphere (http://wdc.kugi. kyoto-u.ac.jp/aedir/index.html, last access: August 2018). The upper (AU) and lower envelope (AL) of the superposed plots of all the data from these stations are used in this study as functions of UT.

Further, we employ the SymH and AsyH indices, which describe the geomagnetic disturbances at midlatitudes in terms of longitudinally asymmetric (ASY) and symmetric (SYM) disturbances for the $\mathrm{H}$ component (http:// wdc.kugi.kyoto-u.ac.jp/aeasy/index.html, last access: August 2018 or, alternatively, https://omniweb.gsfc.nasa.gov/ ow_min.html, last access: August 2018). SymH is essentially the same as the Dst index, but with a different time resolution (1 min cadence).

Finally, Newell et al. (2007) proposed a new solar wind coupling function, representing the rate of magnetic flux opened at the magnetopause, $d \Phi_{\mathrm{MP}} / \mathrm{d} t$, which is referred to here as Index N (IndN). It is used for the correlation analysis in the solar-terrestrial physics and is expressed as follows;

IndN $=d \Phi_{\mathrm{MP}} / \mathrm{d} t=v^{4 / 3} B_{T}^{2 / 3} \sin ^{8 / 3}\left(\theta_{\mathrm{c}} / 2\right)$.

Here, $\Phi$ (or $\Phi_{\mathrm{PC}}$ ) is the (open) magnetic flux that constitutes the polar cap, $v$ describes the solar wind speed, or more precisely, the transport velocity of IMF field lines that approach the magnetopause. $B_{T}$ is the magnitude of the IMF, $\sin ^{8 / 3}\left(\theta_{c} / 2\right)$ is the percentage of field lines, which subsequently merge, and the IMF clock angle $\theta_{\mathrm{c}}$ is defined by $\theta_{\mathrm{c}}=$ $\arctan 2\left(B_{y} / B_{z}\right)$. This function best describes the interaction between the solar wind and the magnetosphere over a wide variety of magnetospheric activity. IndN has a strong correlation with other indices that characterize both the plasma and the IMF in the solar wind as well as the processes in the magnetosphere. By means of a statistical study of the electro- jet characteristics, the new function IndN was used together with the classical indices SymH, AsyH, and AL. For the determination of all indices throughout this study we used time averages of the overflight intervals.

\section{Method}

Ionospheric currents at high latitudes, which are recorded by low-Earth orbiting (LEO) satellites as magnetic field deviations, represent the sum of FACs between the magnetosphere and ionosphere (Birkeland currents) and predominantly horizontal ionospheric currents, which flow mainly in the highly conducting ionospheric $\mathrm{E}$ layer below the satellite orbit.

The horizontal sheet currents are commonly decomposed in two different ways. The classical fundamental theorem of vector calculus, known as Helmholtz's theorem, states that any vector field can be decomposed into the sum of a curlfree and a divergence-free part. On the other hand, considering the relation to an electric field, the sheet current is composed of Pedersen currents, which flow in the direction transverse to the magnetic field and parallel to the electric field in the neutral wind frame of reference (e.g. Richmond, 1995; Baumjohann and Treumann, 1996), and Hall currents, which are perpendicular to both fields. The latter decomposition requires, however, the knowledge of the electric field in the neutral wind frame of reference, which is not given in our case.

Using the Helmholtz theorem, we assume the Hall currents to be divergence-free, i.e. they are supposed to close entirely within the ionosphere, while the Pedersen currents are curl-free, connecting essentially various branches of FACs. (Laundal et al., 2018) showed (see their Fig. 14) that during summer conditions, which is the case for the six storm intervals analyzed in this study, the divergence-free and curl-free ionospheric currents are mainly represented by the Hall and Pedersen currents, respectively.

The Hall current at high latitudes are derived from CHAMP scalar magnetometer records along the satellite orbits according to the method that was presented by Ritter et al. (2004b). This method of Hall current estimation from scalar magnetometer records of satellites was proposed for the first time by Olsen (1996). These calculations make use of a current model consisting of a series of 160 infinite cur- 
rent lines at an altitude of $110 \mathrm{~km}$ and separated by $1^{\circ}$ in latitude. The magnetic field of the line currents were related to the current strength $I$ according to the Biot-Savart law. The strength of each of the 160 current lines were derived from an inversion of the observed field residuals using a leastsquares fitting approach. The model does not take into account the contributions from field-aligned and Pedersen currents, measured at CHAMP altitudes. The comparison with ground-based geomagnetic variations of the horizontal component that considers only the contributions from the ionospheric Hall current field, because the contributions from the field-aligned and the Pedersen currents largely cancel each other out (Fukushima, 1976), showed the applicability of the modelling assumptions by Ritter et al. (2004b) with high reliability, particularly for the estimation of the Hall currents.

The level of ionization in the near-noon hours at latitudes of $75^{\circ}<\Phi<80^{\circ}$ decreases from the summer to winter season by about 1 order of magnitude (Feldstein et al., 1975a). The PE current strength amounts to $\sim 0.1 \mathrm{~A} \mathrm{~m}^{-1}$ during winter, which makes it difficult to be measured adequately by magnetometers aboard satellites. Because of that we investigate only summer storms in this study: three storms with CHAMP orbits in the midday-midnight plane and three in the dawn-dusk plane (listed in Table 1). Here, we describe only one of these storms, namely that of 29 May-30 May 2003. The five other storms are described in the Appendix of this paper.

The storm phases are identified in this study according to the SymH index, which describes, together with the AsyH index, the large-scale variations of the geomagnetic field with a 1 min cadence. In essence, SymH represents the mean value of the magnetic field deviation from the quiet-time level for a longitudinally distributed chain of six midlatitude stations.

The intensity of the ring current varies with longitude. This variability, denoted by the AsyH index, is determined as the range between the maximum and minimum magnetic field values of the disturbance field minus the SymH from the longitudinal chain of midlatitude stations.

\section{The storm on 29-30 May 2003}

The orbit of the CHAMP satellite in its ascending branch was on the dayside ( 14:00-16:00 MLT), while its descending branch was in the nighttime sector ( 02:00-04:00 MLT). Figure 1 shows various geomagnetic indices and the variation of the $B_{y}$ and $B_{z}$ components of the IMF during the storm progression together with the times of CHAMP satellite observations during crossings of the northern polar cap regions, indicated by dashed vertical lines. The respective CHAMP orbit numbers are given above the uppermost panel, with the first three digits on the left side and the last two digits in between the two vertical dashed lines that indicate the corresponding observation intervals.
The beginning of the main magnetic storm phase was identified during orbit 16233 at 22:24 UT, with an average SymH value of $-61.6 \mathrm{nT}$ for the overflight interval, while the minimum value of $\mathrm{SymH}$ was recorded during orbit 16234 at 23:59 UT $(-123.5 \mathrm{nT})$ and orbit 16235 at 01:33 UT $(-139.5 \mathrm{nT})$. The four orbits prior to the main phase (1622916232) at 16:18-20:53 UT are characterized by the SymH values of $-1.6,-35.6,-59.6$, and $-27.0 \mathrm{nT}$ as well as the occurrence of three substorms with intensities according to $\mathrm{AL}$ values in the range of $\sim-1600$ to $\sim-2400 \mathrm{nT}$. AsyH increases sharply prior to the beginning of the main phase (208.3 nT during orbit 16231) and during the beginning of the main phase (290.7 nT during orbit 16233). In the maximum of the main phase, the values of this index decrease to $75.5 \mathrm{nT}$ during orbit 16234 and $145.0 \mathrm{nT}$ during orbit 16235 . Following the main phase, the recovery phase develops (orbits 16236-16240) at 03:03-09:21 UT, in the course of which the SymH values return to the initial values at $\sim-60 \mathrm{nT}$, and AsyH decreases to $51 \mathrm{nT}$ during orbit 16239.

Let us now consider the structure and the latitudinal variation of the position and strength of the electrojet during the various phases of the analyzed storm. The eastward (EE) and westward electrojet (WE) can exist in the daytime sector at latitudes of the auroral zone $\left(\sim 60^{\circ}<\right.$ MLat $\left.<70^{\circ}\right)$, while poleward of it, at latitudes of the auroral oval $(\sim$ $73^{\circ}<$ MLat $<79^{\circ}$ ), the currents of the polar electrojet $(\mathrm{PE})$ can appear. The direction of the PE, however, can be eastward or westward. This is determined by the sign of the IMF $B_{y}$ component; the eastward current in the PE is $B_{y}>0$ and the westward is $B_{y}<0$. In the nighttime sector, the current is directed westward (WE) in the majority of cases at auroral latitudes. Figure 2 shows the direction, MLat, and strength of the Hall currents along the orbit for dayside (left column) and nightside (right column) sectors as obtained from the scalar measurements of the geomagnetic variations corresponding to the modelled current variations of Ritter et al. (2004b). The current direction is related to the orientation of the satellite orbit; positive currents point eastward for the descending orbital parts and westward for the ascending. The auroral electrojet current flow is assumed to be in a strict eastwest direction due to method constraints. It is obvious that the quantity, locations, and intensity of the peaks along the latitudinal current intensity distribution vary in the course of the storm development. A close correlation of the EE with the WE can be expected for the AU and AL indices, respectively, which are regarded as a measure of the auroral electrojets from the ground. We are considering, however, magnetic records along the satellite orbit well above the ionospheric current layer.

\subsection{Observations related to SymH variations}

The latitudinal variation of the position and strength of the $\mathrm{EE}$ is shown in Fig. 2 in the left panels. During the orbits 16229 and 16230, one singular peak of the eastward 
29-30 May 2003

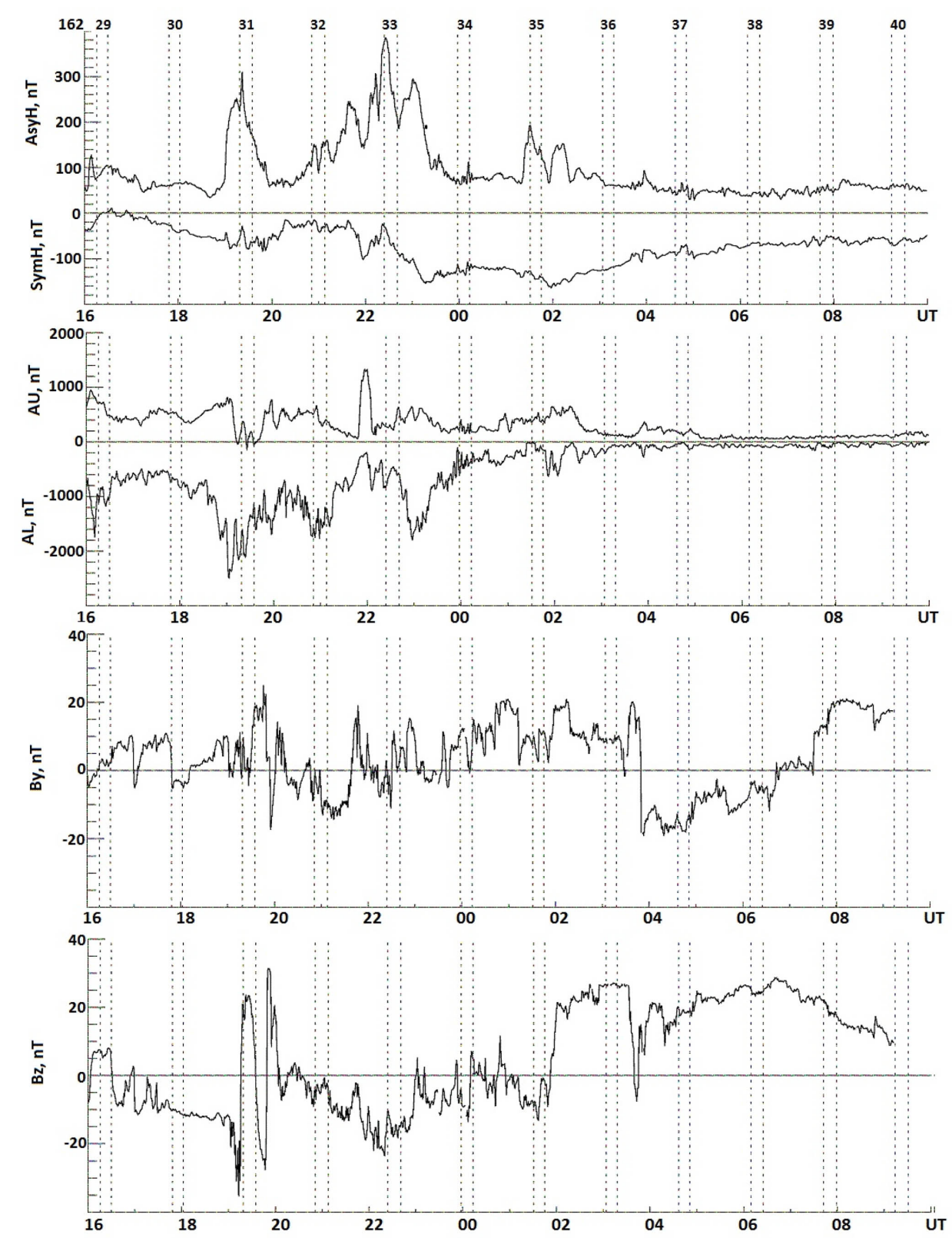

Figure 1. One-minute values of the AsyH, SymH, AU, and AL indices and of the $B_{y}$ and $B_{z}$ components of the IMF for the storm on 29-30 May 2003 (analyzed interval from 16:00 UT on 29 May to 10:00 UT on 30 May 2003, orbits 16229-16240). The paired vertical dashed lines indicate the UT time intervals of each satellite crossing over the northern polar cap. The orbit numbers are split into two parts; the two digits above the uppermost frame denote the last two digits of the orbit numbers of the CHAMP passes, while the first three digits are indicated at the upper left side.

current was observed, which occurred at MLat $=63.4^{\circ}$ and MLat $=64.0^{\circ}$ with intensities of 1.0 and $0.6 \mathrm{~A} \mathrm{~m}^{-1}$, respectively. This means that the EE peak current diminishes in intensity, with increasing disturbances according to the $\mathrm{SymH}$ index and shifts to higher latitudes. During the orbits 1623116233 (in the substorm interval and at the beginning of the main storm phase), one can clearly note two intense peaks: one of the eastward current (EE) and another of the westward current (WE - the westward electrojet). The EE peak during orbit 16231 amounts to $\sim 1.83 \mathrm{~A} \mathrm{~m}^{-1}$ at MLat $=56.3^{\circ}$, decreasing in the course of the next orbit to $0.94 \mathrm{~A} \mathrm{~m}^{-1}$ at MLat $=63.9^{\circ}$. 
(a)

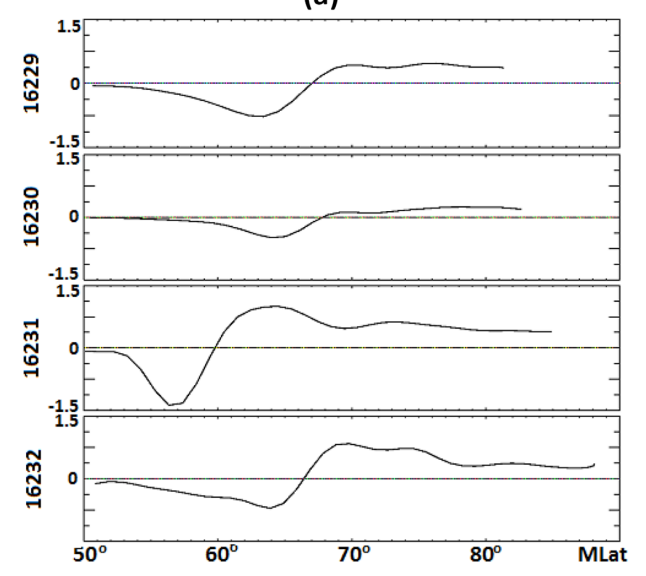

Main phase
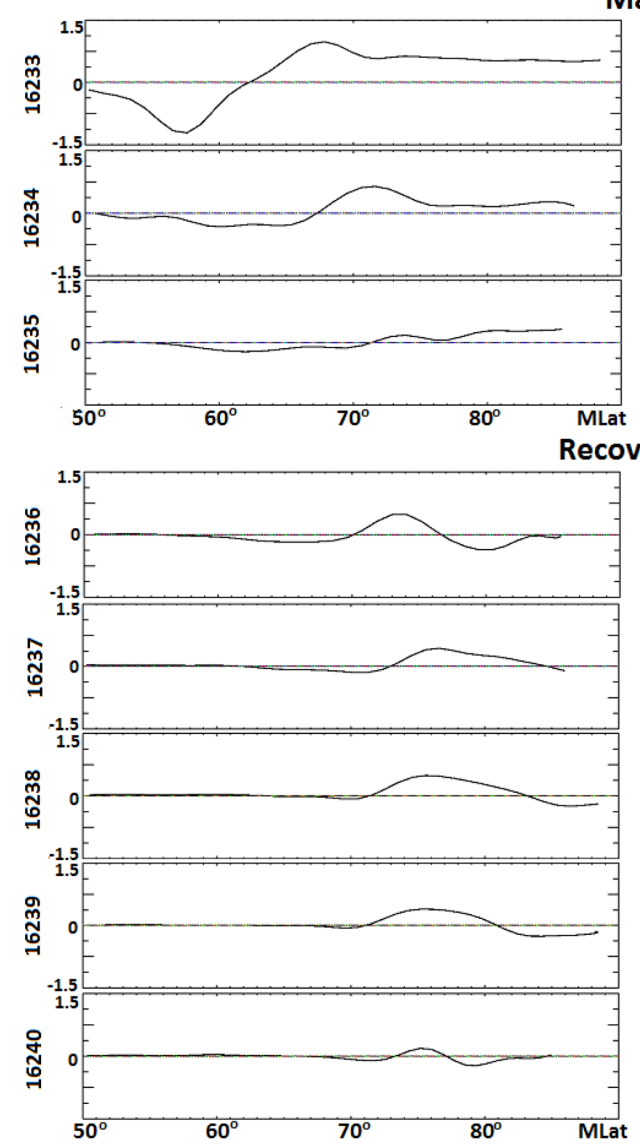

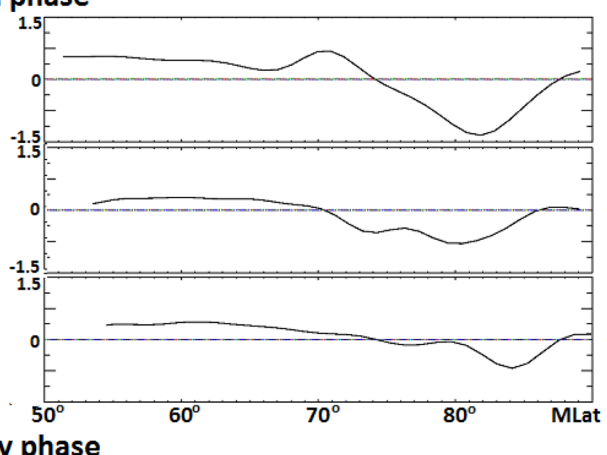

(b)
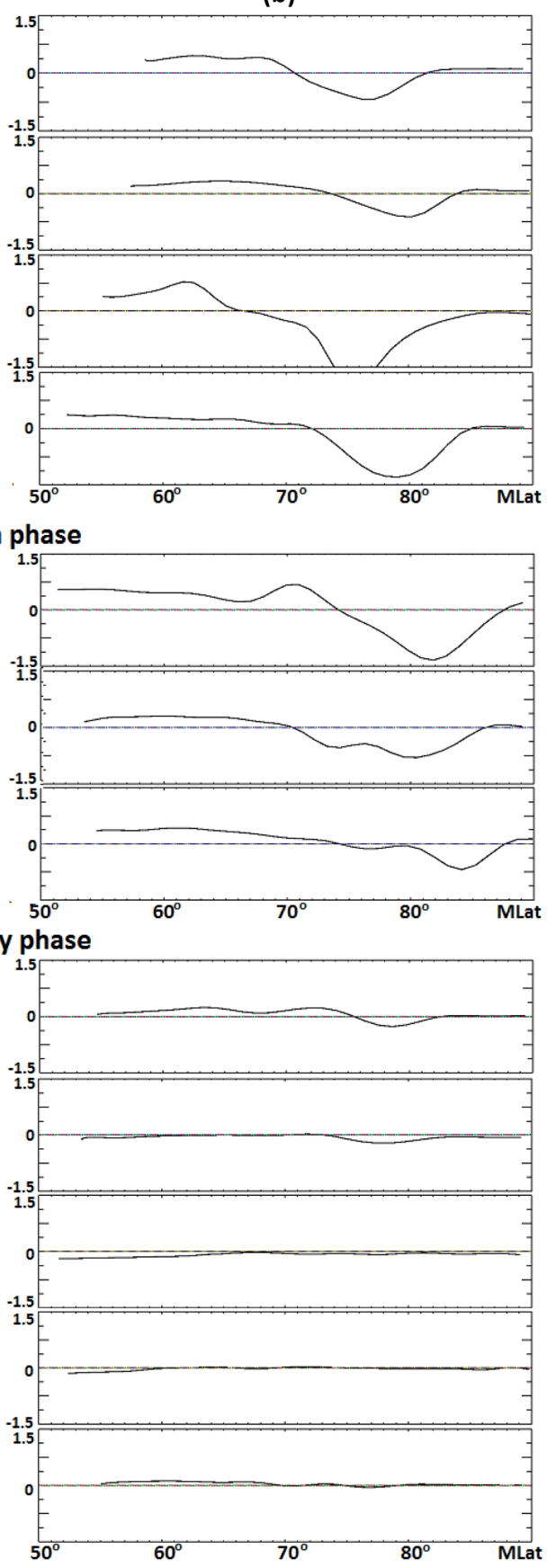

Figure 2. Direction and intensity values of the Hall current in units of ampere per meter $\left(\mathrm{A} \mathrm{m}^{-1}\right)$ along the satellite orbit at the dayside (a, 14:00-16:00 MLT, corresponding to the ascending section of the orbit) and nightside sectors (b, 02:00-04:00 MLT, descending orbit section). Positive currents denote an eastward current for the descending orbit section and a westward current for the ascending section, respectively.

With the beginning of the main phase, the current again intensifies to $1.61 \mathrm{~A} \mathrm{~m}^{-1}$ at MLat $=57.6^{\circ}$. The EE variations in strength and latitudinal position during the orbits 1623116233 proceed analogous to the SymH changes; the more intense SymH, the closer to the equator shifts the EE peak becomes and the stronger its current intensity.
But such an accordance is broken again in the maximum of the main phase similar to orbits 16234 and 16235; SymH increases in intensity, while the $\mathrm{EE}$ in its peak diminishes to $0.44 \mathrm{~A} \mathrm{~m}^{-1}$ and even $0.29 \mathrm{~A} \mathrm{~m}^{-1}$ at MLat $=61.0^{\circ}$. The EE peak amounts to $0.23 \mathrm{~A} \mathrm{~m}^{-1}$ at MLat $=65.2^{\circ}$ during orbit 16236 in the recovery phase and diminishes further to val- 
ues $<0.2 \mathrm{~A} \mathrm{~m}^{-1}$ during the subsequent orbits, which complicates the identification of the EE position. This way the EE follows, with its varying current densities at auroral latitudes, the growth (or creation) and main phases of the magnetic storm. It should be noted that SymH still remains significant during the recovery phase, with values of $\sim-60 \mathrm{nT}$. This exceeds the intensity of SymH during the substorm interval and at the beginning of the storm main phase, while the EE during the recovery phase is much smaller than during the substorm interval.

In contrast to the EE, a westward current exists on the dayside sector during the whole interval considered, except the two first orbits, and achieves values of $1.1-1.3 \mathrm{~A} \mathrm{~m}^{-1}$ within the peaks at $64^{\circ}<$ MLat $<70^{\circ}$ during the substorm interval. It is obvious that the WE does not attain the CHAMP meridian ( 14:00-16:00 MLT) during the first two orbits, while it strengthens in the nighttime sector and propagates toward the evening hours. During the orbits 16231-16233, the latitude and strength of the WE peak changes in phase with the SymH intensity, analogous to the current variations in the EE peak. During the maximum of the main phase the WE peak diminishes to $0.8-0.25 \mathrm{~A} \mathrm{~m}^{-1}$ at $71^{\circ}<\mathrm{MLat}<74^{\circ}$ in antiphase to SymH.

The peaks of the westward current remain at a level of $\sim 0.6 \mathrm{~A} \mathrm{~m}^{-1}$ within $73^{\circ}<$ MLat $<76^{\circ}$ during the recovery phase. An additional peak of eastward current with a strength of $0.49 \mathrm{~A} \mathrm{~m}^{-1}$ appears during orbit 16236 at MLat $=80.5^{\circ}$ during early afternoon hours (MLT $\sim 14.5 \mathrm{~h}$ ). This latitude and the MLT range around midday imply that the current observed is the PE. In this case its orientation is controlled by the IMF $B_{y}$ component, and for an eastward PE, the $B_{y}$ component should be positive (Friis-Christensen et al., 1972; Sumaruk and Feldstein, 1973; Feldstein, 1976). Indeed, according to Fig. 1, $B_{y}=9 \mathrm{nT}$ during the period of this orbit. During the two subsequent orbits, the Hall current changes its direction to westward at MLat $\sim 80^{\circ}$. If this westward current proves to be the PE, then its appearance should be connected with a change in the IMF $B_{y}$ component. Indeed, the currents are accompanied with a change in sign of the IMF $B_{y}$ component, corresponding to $-17.5 \mathrm{nT}\left(\mathrm{MLat}=80.7^{\circ}\right)$ during orbit 16237 and $-5.2 \mathrm{nT}\left(\mathrm{MLat}=80.6^{\circ}\right)$ during orbit 16238. During the orbits 16239 and 16240, the IMF $B_{y}$ component turns to positive values again, and weak directed eastward currents appear accordingly at MLat $\sim 80^{\circ}$.

In the majority of the latitudinal profiles in the nighttime sector (Figs. 2, right panels), one peak of the WE exists at latitudes of the auroral oval and some weakly spread eastward currents. Two orbits constitute an exception - one prior to (16231) and another during the beginning of the main storm phase (16233). These orbits pertain to the period of intense substorms. Within the polar cap up to the geomagnetic pole, quite intense (up to $0.9 \mathrm{~A} \mathrm{~m}^{-1}$ ) eastward currents exist.

These currents might contain irregularities, which are caused by the appearance of a peak of eastward currents in the latitudinal profile. The monotonicity of the eastward cur- rent variations within the polar cap during most orbits provides some reason to assume that these currents result from the closure of an intense WE current, which occurs at latitudes of the auroral oval in the nighttime sector.

At the beginning of the substorm interval (orbits 16229 and 16230) with the intensification of SymH, the WE peak shifts to lower latitudes, and the current intensity diminishes. The most intense peaks of the nighttime WE are obtained during the substorm interval prior to the main phase and start with and retain values of $2.7 \mathrm{~A} \mathrm{~m}^{-1}$ at MLat $=64.4^{\circ}$ (orbit 16231), $1.7 \mathrm{~A} \mathrm{~m}^{-1}$ at MLat $=60.9^{\circ}$ (orbit 16232), and during the beginning of the main phase, they start with $1.79 \mathrm{~A} \mathrm{~m}^{-1}$ at MLat $=58.0^{\circ}$ (orbit 16233). Later in the maximum of the main storm phase, the WE peak current strength diminishes to $1.07 \mathrm{~A} \mathrm{~m}^{-1}$ at MLat $=59.5^{\circ}$ (orbit 16234) and $0.9 \mathrm{~A} \mathrm{~m}^{-1}$ at MLat $=55.8^{\circ}$ (orbit 16235). Hence, the latitudinal peaks of the WE vary during nighttime in phase with the intensification of SymH (storm development) before the main phase commences at higher latitudes, while shifting to the equator during the maximum of the main phase. The peak intensities change both in phase and in antiphase with the SymH intensity. During the recovery phase, the peak intensity of the WE current is smaller than $0.2 \mathrm{~A} \mathrm{~m}^{-1}$, while the eastward currents within the polar cap are too small to be recorded.

\subsection{Observations related to AsyH variations and to high-latitude currents}

In the dayside sector during the existence of the EE (orbits 16229-16236), the peak current intensities and the peak latitude positions vary synchronously with the AsyH changes, except for one orbit (16235) during the main phase. During this orbit, the AsyH index abruptly intensifies to $145 \mathrm{nT}$, with a correspondingly small intensity of the EE with $0.29 \mathrm{~A} \mathrm{~m}^{-1}$ and a shift of MLat by $1.8^{\circ}$. For the WE, the change in latitude and strength of the peak currents is in phase with the AsyH variations during the storm, with the exception of orbit 16235.

In the nighttime sector, the intensity of the peaks and their latitude (except orbit 16235) change in phase with the AsyH variations.

\subsection{Summary of the observations}

Summarizing the results of Hall current observations by the CHAMP satellite during the magnetic disturbance period of 29 May-30 May 2003 in the daytime and nighttime sectors (12:00-16:00 and 00:00-04:00 MLT, respectively), we come to the following conclusions:

- Intense $>1 \mathrm{~A} \mathrm{~m}^{-1}$ eastward and westward electrojets can occur at latitudes of the auroral zone during substorm periods, which precede the magnetic storm, and during the beginning of its main phase. During the maximum of the main phase, the strength of the Hall cur- 
rents as well as the substorms diminish in antiphase, with an increase in the SymH index.

- A fast decay of the EE and WE occurs during the recovery phase at auroral latitudes both during daytime and nighttime hours. The westward or the eastward currents can be influenced during this storm phase by the existence of a $\mathrm{PE}$ at $73^{\circ}<\mathrm{MLat}<80^{\circ}$ in the region of the dayside cusp.

- The direction of the current in the PE is determined by the IMF $B_{y}$ component; for $B_{y}>0$ the current is eastward and for $B_{y}<0$ it is westward. The change in the current direction within the PE can occur several times during the storm development, but always in accordance with the change in the IMF $B_{y}$ orientation.

- The Hall currents in the auroral ionosphere, both the $\mathrm{EE}$ and the WE, usually vary in phase with the SymH and AsyH variations (but sometimes also in antiphase). There are time intervals where any correlation between the geomagnetic activity indices and the Hall current parameters is missing (see Tables 2 and 3 below in Sect. 5). There is a closer connection of the current intensity to the MLat variations with AsyH than to SymH.

- In the daytime sector (14:00-16:00 MLT) during a period of intense substorms, the $\mathrm{EE}$ is located in a latitude range $56^{\circ}<\mathrm{MLat}<64^{\circ}$, while the $\mathrm{WE}$ is at $64^{\circ}<$ MLat $<70^{\circ}$. During the main phase of the storm, the EE shifts to $58^{\circ}<\mathrm{MLat}<62^{\circ}$, while the $\mathrm{WE}$ is situated at $64^{\circ}<$ MLat $<73^{\circ}$, and finally during the recovery phase, the $\mathrm{WE}$ is observed at latitudes of $73^{\circ}<$ MLat $<76^{\circ}$. Therefore, the EE stays at about the same latitudes during the both the intense substorms and the main phase of the storm, attaining extreme equatorward values of MLat $\sim 56^{\circ}$. An analogue situation exists with regard to the change in position for the $\mathrm{WE}$ in various storm phases, but during daytime hours the WE is located about $6^{\circ}$ closer to the pole.

- In the nighttime sector (02:00-04:00 MLT), practically only the WE exists, which is located during substorms at $61^{\circ}<$ MLat $<64^{\circ}$ and during the main storm phase at $56^{\circ}<$ MLat $<60^{\circ}$. Therefore, extreme positions of the $\mathrm{WE}$ and EE can reach latitudes below $60^{\circ}$. This occurs in the daytime sector for the EE and in the nighttime for the WE.

The detailed description of the Hall current characteristics during five further magnetic summer storm intervals is transferred to the Appendix.

\section{Correlation analyses and discussion}

In the Sect. 4 and in Appendix A1-A5, we have investigated several geomagnetic storm periods based on magnetometer
Table 2. Correlations of the PE current system with various indices. The columns show the dependent $(X)$ and independent variable $(Y)$, their correlation coefficients $(r)$, the coefficients $A$ and $B$ of the regression equations $X=A+B \times Y$, and their dispersions $\sigma$.

\begin{tabular}{lrrrrr}
\hline$X$ & $Y$ & $r$ & $A$ & $B$ & $\sigma$ \\
\hline$I$ (intensity, $\left.\mathrm{A} \mathrm{m}^{-1}\right)$ & $B_{y}(>0)$ & 0.59 & 0.535 & 0.018 & 0.160 \\
$I$ (intensity, $\left.\mathrm{A} \mathrm{m}^{-1}\right)$ & $B_{y}(<0)$ & -0.72 & 0.291 & -0.024 & 0.134 \\
$I$ (intensity, $\left.\mathrm{A} \mathrm{m}^{-1}\right)$ & $\left|B_{y}\right|$ & 0.56 & 0.433 & 0.018 & 0.170 \\
$I$ (intensity, $\left.\mathrm{A} \mathrm{m}^{-1}\right)$ & $\mathrm{AsyH}$ & 0.74 & 0.396 & 0.004 & 0.138 \\
MLat (deg.) & $\mathrm{AL}$ & 0.46 & 78.540 & 0.006 & 2.542 \\
MLat (deg.) & $\mathrm{IndN}$ & -0.52 & 77.750 & -0.006 & 2.415 \\
\hline
\end{tabular}

measurements onboard the CHAMP satellite. The Hall currents in the high-latitude upper ionosphere of the Northern Hemisphere were analyzed for various MLT sectors with regard to their position in geomagnetic latitude, their strength, and their direction. The empirical description concerned the appearance of the EE, the WE, and the PE during various storm phases and was carried out primarily qualitatively.

Below we are going to analyze the current directions, their densities, and MLat positions for various MLT sectors with regard to solar wind parameters and some indices of the planetary magnetic activity (SymH, AsyH, AL, and IndN). We use activity indices, which characterize the occurrence and dynamics of the large-scale plasma domains in Earth's magnetosphere that are responsible for the existence of concrete variations in the geomagnetic field at Earth's surface.

\subsection{Polar electrojets}

It is well known from geomagnetic activity research that the intense magnetic disturbances at the high-latitude projection of the magnetospheric cusp are not related to the occurrence and dynamics of magnetospheric substorms.

Figure $3 \mathrm{a}-\mathrm{f}$ shows the correlations of the various IMF parameters and geomagnetic indices, with the magnetic latitude (left side panels) and the Hall current intensity $I$ (right side panels) obtained by CHAMP satellite crossings over the polar electrojets during six geomagnetic storms. The direction of the Hall currents can be distinguished in the upper panels (Fig. 3a); westward and eastward currents are indicated with blue and red data points, respectively. For further study, we selected the electrojet parameters at their extremal values of the current strength for each orbit. The data pool was augmented, yet also includes neighbouring values before and after the extremal points.

Figure 3 a differentiates the current measurements with regard to the azimuthal IMF component $\left(B_{y}\right)$, i.e. between those obtained during $B_{y}>0$ and those obtained during $B_{y}<0$ conditions. It is clearly seen that the direction of the current within the PE is determined by the IMF $B_{y}$ sign. For intervals with positive IMF $B_{y}>0$, we observe a Hall current directed eastward; for negative IMF $B_{y}<0$ intervals the Hall current is always westward. The current strength within 
PE peak location

(a)

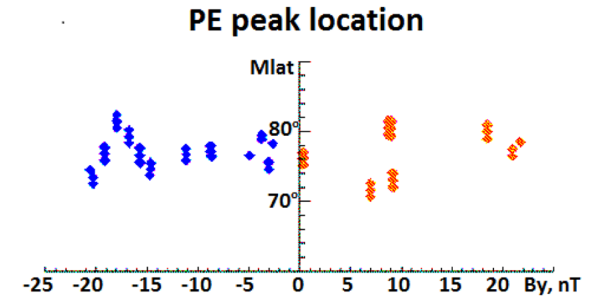

Peak current sheet density
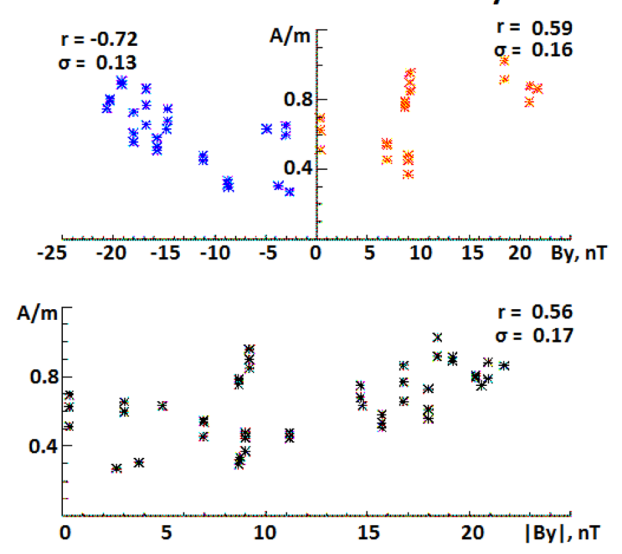

(c)
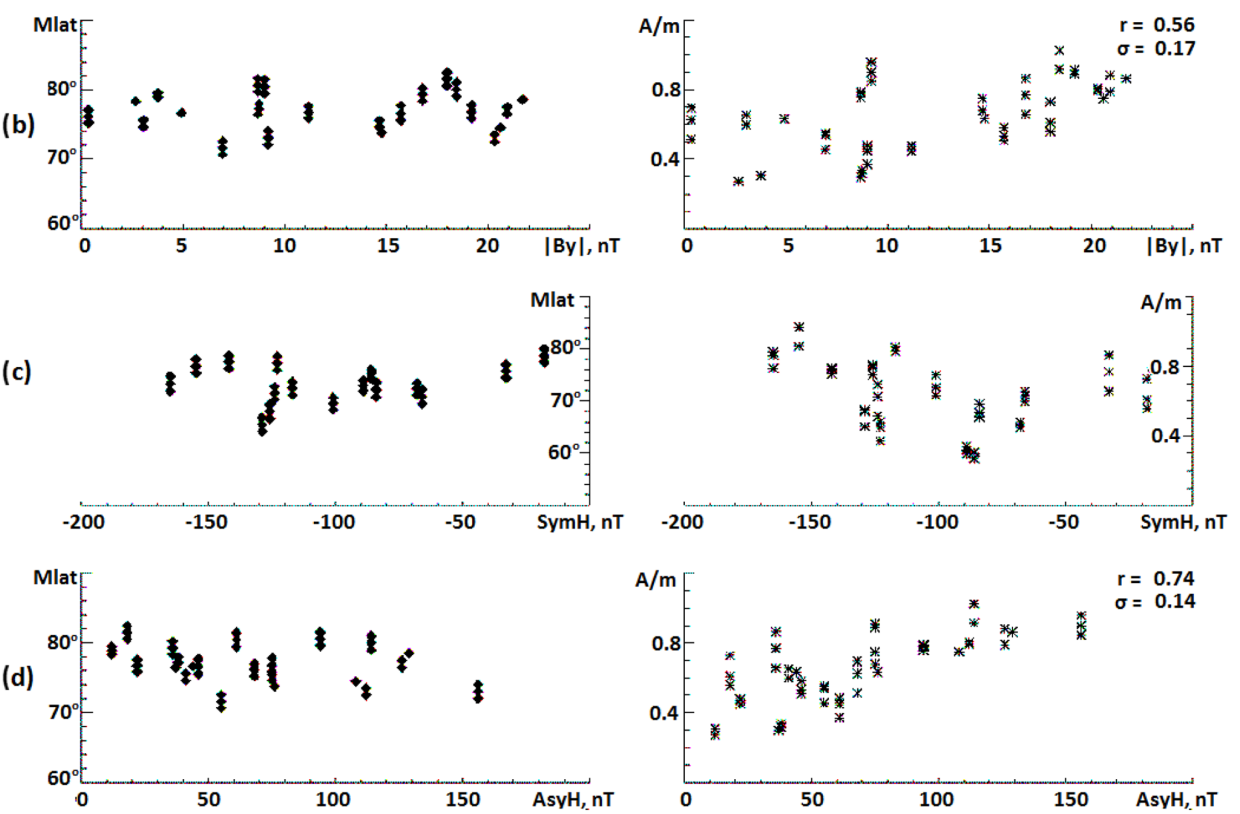

$$
\begin{aligned}
& r=0.46 \\
& \sigma=2.54
\end{aligned}
$$

(e)
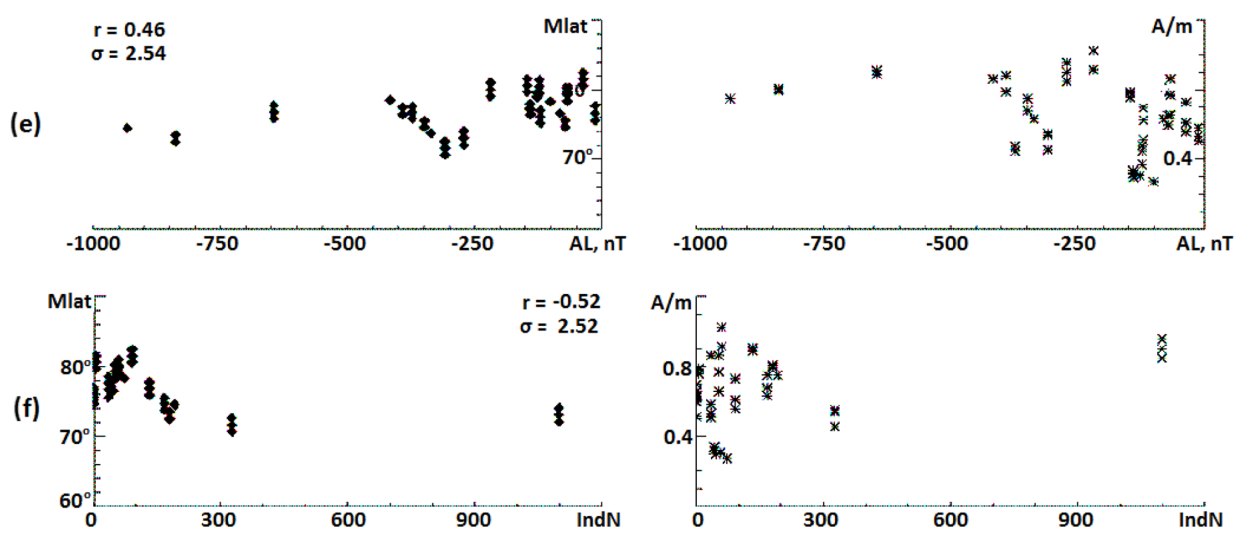

Figure 3. Dependence of the magnetic latitude MLat (degrees) position at the peak (left column) and the intensity $\left(I\right.$ in $A m^{-1}$, right column) in the Hall current of the polar electrojet (PE) on the IMF $B_{y}$ component (a) and its magnitude (b) in the geomagnetic activity indices SymH (c), AsyH (d), AL (e), and the solar wind coupling function IndN (f). The blue and red data points indicate westward and eastward currents, respectively. For the cases of correlation with $r>0.46$, the correlation coefficients $(r)$ and the dispersion $(\sigma)$ according to a linear regression are shown as labels.

the PE is correlated with the magnitude of the azimuthal IMF component $B_{y}$; it increases from $0.3-0.4 \mathrm{~A} \mathrm{~m}^{-1}$ for near-zero values to $I \sim 0.9 \mathrm{~A} \mathrm{~m}^{-1}$ for $\left|B_{y}\right| \sim 23 \mathrm{nT}$ (i.e. the maximum $B_{y}$ value during the periods investigated). Correlation coefficients $r$ between current intensity $I$ and the IMF $B_{y}$ component for $B_{y}>0$ and $B_{y}<0$ are shown in the upper right and upper left corner, respectively (Fig. 3a, right panel). The coefficients for the offset $(A)$ and the slope $(B)$ of the linear regression line (for $r \geq 0.46$ ) as well as the dispersion values $\sigma$ are listed in Table 2.

The current intensity values $I$ for $B_{y} \sim 0 \mathrm{nT}$ are somehow different in the regression equations for $B_{y}>0$ and 
$B_{y}<0$. Figure $3 \mathrm{~b}$ (right panel) shows the current intensity $I$ as a function of the IMF component's magnitude $\left|B_{y}\right|$, i.e. independent of the IMF $B_{y}$ sign. This increases the number of data points for the linear regression estimation. According to this estimation, the current intensity amounts to $I \sim 0.4 \mathrm{~A} \mathrm{~m}^{-1}$ for $\left|B_{y}\right| \approx 0 \mathrm{nT}$, while it attains $\sim 0.9 \mathrm{~A} \mathrm{~m}^{-1}$ for $\left|B_{y}\right|=23 \mathrm{nT}$, i.e. about twice as large. The increase in the Hall current strength within the PE can occur also in magnetically quiet intervals during the absence of magnetic activity at latitudes of the auroral zone, because such disturbances are not directly related to the IMF $B_{y}$ component.

We could not find any essential correlation between the MLat position and the IMF $B_{y}$ component, neither for both $B_{y}>0(r=0.04)$ and $B_{y}<0(r=0.32)$ nor for $\left|B_{y}\right|(r=$ 0.15 , see Fig. 3a and b). The current strength and its direction (eastward or westward) within the PE is controlled by the IMF $B_{y}$ component, but the latitudinal position of the current intensity maximum does not depend on IMF $B_{y}$. The observed morphological peculiarity of the PE is caused by its generation mechanism. This is assumed to be due to the interaction between the magnetosphere and the supersonic plasma flow (solar wind) with a "frozen-in" magnetic field (IMF). The PE currents are generated at magnetic latitudes of the cusp due to reconnection processes between the IMF and the geomagnetic field (Wilhjelm and Friis-Christensen, 1971; Jørgensen et al., 1972). The reconnection of magnetic fields brings about a north-south electric field and an east-west Hall current at cusp latitudes in the ionosphere.

The SymH index varied during the intervals of CHAMP overflights above the polar electrojets considered in this study, from -10 to $-170 \mathrm{nT}$. As shown in Fig. 3c, there is no correlation between SymH and the MLat positions $(r=-0.13)$ nor the PE current strength $(r=-0.01)$. The absence of any correlation is as expected, because the current system of the ring current DR(sym) is located completely within the magnetosphere. In case of absent FACs, it cannot serve as a source for Hall currents in the ionosphere that is responsible for the existence of $\mathrm{PE}$.

According to Fig. 3d, there is a high correlation between the AsyH index and the PE current $I(r \sim 0.74)$ and an absence of correlation with the MLat position of the PE $(r \sim-0.29)$. The PE current strength has therefore a direct relation to the intensity of the AsyH current system; increasing longitudinal asymmetry increases the PE current intensity, but the latitudinal position of the PE does not depend on AsyH.

A partial ring current (PRC) emerges during geomagnetic disturbances. The PRC is a current directed westward in the equatorial plane of the evening to nighttime sector at geocentric distances of $R \sim 3-4 R_{\mathrm{E}}$. The basic PRC current system includes Region-2 FACs into the ionosphere within this sector (Iijima and Potemra, 1976a) and an EE in the ionosphere (Feldstein et al., 2006; Kalegaev et al., 2008). Observations of fluxes of energetic neutral atoms (ENA) show increased ion fluxes in the evening-nighttime sector within the inner magnetosphere during geomagnetic disturbances, which appears to be the experimental evidence for the existence of the PRC (Kozyra and Liemohn, 2003). A successful modelling of the PRC and the EE has been performed by Kalegaev et al. (2008) for the magnetic storm event on 6 November-14 November 2004.

The high correlation between AsyH and the intensity of the PE gives reason to assume an IMF $B_{y}$ control of the characteristics of the magnetic field asymmetry inside the magnetosphere. This component of the IMF does not directly influence the intensity of the PRC, but it influences via the current system that forms by the PE, with FACs between the PE and the PRC.

The PRC position in the equatorial plane is controlled by MLT, and toward the near-noon sector, it shifts to the ionospheric footpoint of the cusp region (Feldstein et al., 2006). The geocentric distance of the PRC therefore increases in the nighttime via the evening to the earlier hours. FACs of the PRC map from inner-magnetospheric heights to the nearcusp region, where the Hall currents flow that are controlled by the IMF $B_{y}$ component. One may speculate that this way forms a PRC current system, which, in addition to the basic one, is controlled by the IMF $B_{y}$ component. The observed correlation can have also other explanations.

Figure 3e shows the correlation between the MLat position and the strength of the PE with the AL index of geomagnetic activity. The AL index appears to be a sensitive tracer for processes in the central plasma sheet of the magnetospheric tail. These processes are created by the injection of energetic particles, their accumulation, and the dissipation of their energy during storm times, and they are accompanied by changes in the boundary positions of large-scale plasma structures. They appear to have a relatively small influence on the strength and MLat position of the PE (with $r=-0.38$ and $r=0.46$, respectively). However, there is a distinctive tendency for the shift of the PE from $\sim 78$ to $\sim 74^{\circ}$, with an increase in the AL index up to $-900 \mathrm{nT}$.

As shown in Fig. 3f, there is a correlation of IndN with MLat in the daytime sector $(r=-0.52)$. This is obvious, because both components $B_{y}$ and $B_{z}$ are included in the definition of IndN. With increasing IndN, the latitude of the current decreases. The correlation coefficient of IndN with the Hall current intensity $(I)$ is $r=0.3$.

To summarize, the values of the correlation coefficients $r$ and the coefficients $A$ and $B$ of the regression equations are listed in Table 2. They relate the PE current intensity and their MLat position to the indices that characterize the situation in the solar wind and within the magnetosphere at the time of the observations. They are characterized by the following peculiarities:

- The PE appears at the magnetic latitudes and local times of the cusp.

- The direction of the current in the PE is controlled by the IMF $B_{y}$ (azimuthal) component; for $B_{y}>0$ the cur- 
Table 3. The dependent $(X)$ and the independent variable $(Y)$, their correlation coefficients $(r)$, the coefficients $A$ and $B$ of the regression equations $X=A+B \times Y$, and their dispersions $\sigma$, listed for four different MLT intervals.

\begin{tabular}{|c|c|c|c|c|c|}
\hline$X$ & $Y$ & $r$ & $A$ & $B$ & $\sigma$ \\
\hline \multicolumn{6}{|c|}{ MLT 09:00-14:00 } \\
\hline MLat (WE, deg.) & AsyH & -0.54 & 74.136 & -0.052 & 3.89 \\
\hline MLat (EE, deg.) & AsyH & -0.49 & 70.327 & -0.047 & 3.91 \\
\hline MLat (EE, deg.) & AL & 0.68 & 70.192 & 0.005 & 3.28 \\
\hline MLat (WE, deg.) & IndN & -0.74 & 72.215 & -0.011 & 3.13 \\
\hline MLat (EE, deg.) & IndN & -0.67 & 70.271 & -0.025 & 3.43 \\
\hline \multicolumn{6}{|c|}{ MLT 14:00-21:00 } \\
\hline MLat (WE, deg.) & SymH & 0.49 & 72.783 & 0.041 & 4.23 \\
\hline MLat (EE, deg.) & AsyH & -0.54 & 65.971 & -0.036 & 3.67 \\
\hline Intensity (WE, $\mathrm{A} \mathrm{m}^{-1}$ ) & AsyH & 0.68 & 0.168 & 0.003 & 0.24 \\
\hline Intensity $\left(\mathrm{EE}, \mathrm{A} \mathrm{m}^{-1}\right)$ & AsyH & 0.64 & 0.217 & 0.004 & 0.29 \\
\hline MLat (EE, deg.) & AL & 0.46 & 64.707 & 0.004 & 3.88 \\
\hline Intensity $\left(\mathrm{EE}, \mathrm{A} \mathrm{m}^{-1}\right)$ & $\mathrm{AL}$ & -0.59 & 0.320 & -0.001 & 0.31 \\
\hline \multicolumn{6}{|c|}{ MLT 21:00-02:00 } \\
\hline MLat (WE, deg.) & SymH & 0.53 & 63.806 & 0.032 & 2.25 \\
\hline Intensity (WE, $\mathrm{A} \mathrm{m}^{-1}$ ) & AsyH & 0.50 & 0.205 & 0.005 & 0.33 \\
\hline Intensity (WE, $\mathrm{A} \mathrm{m}^{-1}$ ) & $\mathrm{AL}$ & -0.67 & 0.233 & -0.001 & 0.28 \\
\hline Intensity (WE, $\mathrm{A} \mathrm{m}^{-1}$ ) & IndN & 0.76 & 0.207 & 0.003 & 0.25 \\
\hline \multicolumn{6}{|c|}{ MLT 02:00-09:00 } \\
\hline MLat (WE, deg.) & SymH & 0.47 & 67.343 & 0.040 & 3.24 \\
\hline Intensity (WE, $\mathrm{A} \mathrm{m}^{-1}$ ) & AsyH & 0.69 & -0.089 & 0.010 & 0.38 \\
\hline Intensity (WE, $\mathrm{A} \mathrm{m}^{-1}$ ) & $\mathrm{AL}$ & -0.52 & 0.328 & -0.001 & 0.44 \\
\hline
\end{tabular}

rent is eastward, and for $B_{y}<0$ the current is directed westward.

- The current strength in the PE increases with the intensity of the IMF $B_{y}$ component, from $I \sim 0.4 \mathrm{~A} \mathrm{~m}^{-1}$ for $B_{y} \sim 0 \mathrm{nT}$ up to $I \sim 1.0 \mathrm{~A} \mathrm{~m}^{-1}$ for $B_{y} \sim 23 \mathrm{nT}$.

- The MLat position of the PE does not depend on the orientation and the strength of the IMF $B_{y}$ component.

- There is no connection between MLat and the current intensity $I$ in the PE with the magnetospheric ring current (DR, index SymH).

- There is a correlation between the current intensity $I$ in the $\mathrm{PE}$ and the strength of the partial ring current in the magnetosphere (PRC, index AsyH), but there is practically no correlation of this index with the MLat of the PE.

- The currents in the central plasma sheet appear to have a weak influence on the current intensity and the MLat position of the cusp.

- We realized that there is a correlation between MLat and the IndN solar wind coupling function.

\subsection{Auroral electrojets}

The most intense Hall currents at ionospheric heights, which are responsible for the electrojets, are located at auroral latitudes in the nighttime hours. It is there where intense auroras occur most often in the zenith (Chapman and Bartels, 1940; Harang, 1951). These electrojets were named auroral electrojets (AE). A huge number of studies have been published on their morphology, their connections with the solar wind parameters, and the plasma domains in Earth's magnetosphere as well as on their internal processes. The $\mathrm{AE}$ are present during all hours of the day. Based on magnetometer data of the IMAGE and European Incoherent SCATter Scientific Association (EISCAT) networks, Feldstein et al. (1997) showed that the electrojets shift equatorward during the main phase of strong magnetic storms. For DST $\sim-300 \mathrm{nT}$, the EE in the evening and the WE in the nighttime and early morning hours shifts to $\sim 54-\sim 55^{\circ}$. The number of electrojets, their internal current structure, and the interconnection with the individual magnetospheric plasma domains depend both on the activity level and on the MLT position of the observation (Feldstein et al., 2006). Therefore, we consider below the results of the Hall current observations of the CHAMP satellite separately for each of the following four MLT sectors: daytime, nighttime, evening, and morning hours.

Figures 4-7 consider the MLat positions (left columns) and current densities $I$ (right columns) during the moments of extreme values of current strength in dependence on the SymH, AsyH, AL, and IndN indices. As in Fig. 3a, data points of electrojets with an eastward direction are indicated by the red colour and those with westward direction by the blue colour.

Table 3 provides the correlation coefficients $r$, the coefficients $A$ and $B$ of the linear regression equations of the type $X=A+B \times Y$, which were obtained by the least-squares method with correlation coefficients $r>0.46$, and the meansquare deviation $\sigma$ from the regression line.

\subsubsection{Daytime sector 09:00-14:00 MLT}

The AE in the daytime sector can coexist with the PE. These two types of currents can be distinguished according to the following indications (that are valid for AE in contrast to $\mathrm{PE}$ ):

1. The $\mathrm{AE}$ are, as a rule, located at MLat $<73^{\circ}$ during low geomagnetic activity conditions.

2. The Hall current direction in the AE does not depend uniquely on the orientation of the IMF $B_{y}$ component.

Figure 4a shows cases of $\mathrm{AE}$ appearance in the daytime sector with a change in the SymH index. Usually, SymH has negative values $(\mathrm{SymH}<0 \mathrm{nT}$ ) during geomagnetic storms. Figure 4a also shows, however, some values with $\mathrm{SymH}>0$ besides the mostly negative values. They occur as a rule during the first few hours of magnetic storms. The large scatter 


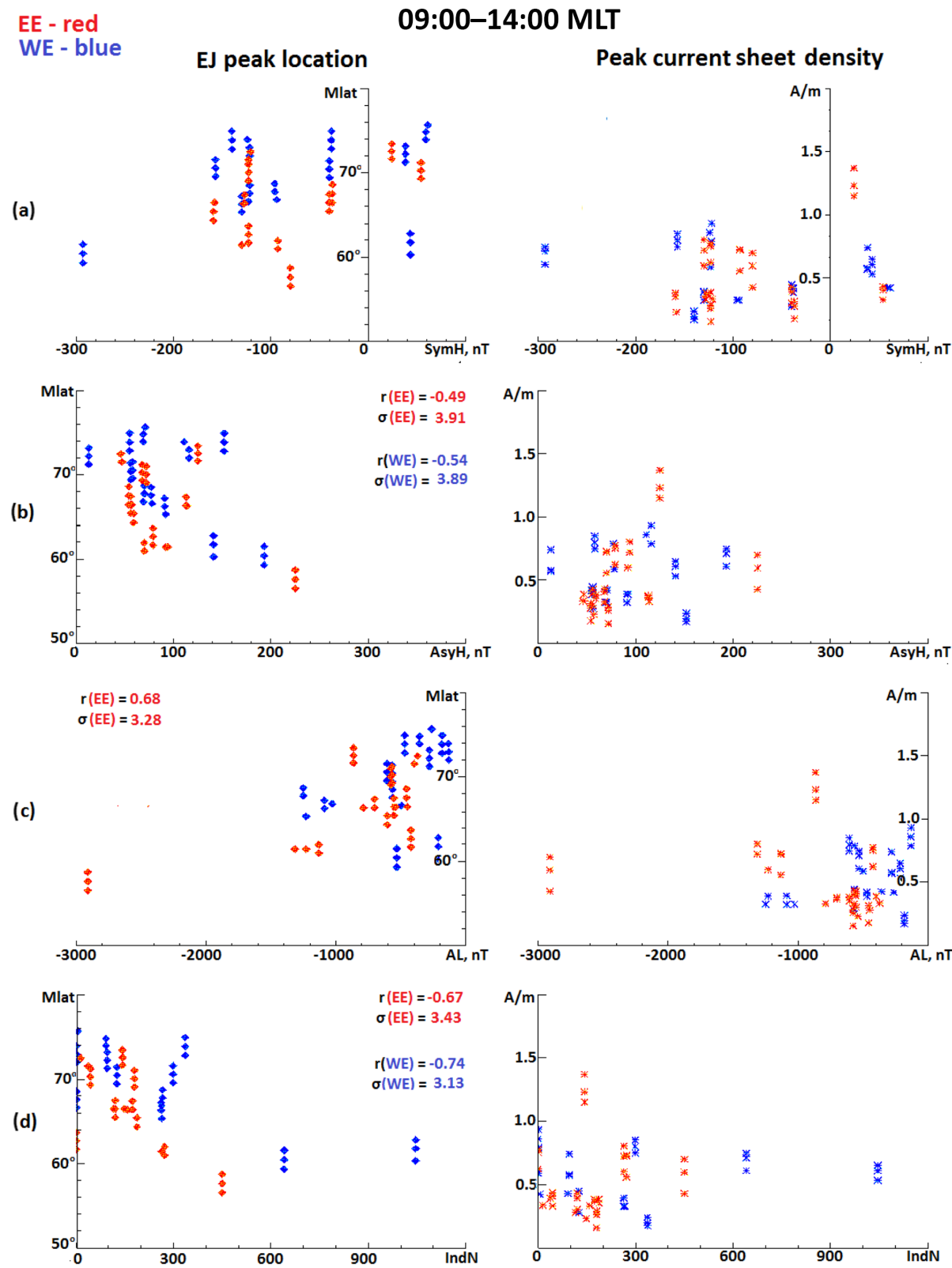

Figure 4. For the daytime sector (09:00-14:00 MLT), the dependence of the magnetic latitude MLat's (degrees) position at the peak (left column) and the intensity ( $I$ in A $\mathrm{m}^{-1}$, right column) of the Hall current in the WE (blue) and EE (red) in the geomagnetic activity indices SymH (a), AsyH (b), AL (c), and the solar wind coupling function IndN (d). For the cases of correlations with $r>0.46$, the correlation coefficients $(r)$ and the dispersion $(\sigma)$ according to a linear regression are shown as labels.

of the data points and their low correlation coefficients (maximum for MLat - EE, $r=0.39-$ and $I-\mathrm{EE}, r=0.29-$ in Fig. 4a) indicate the weak control of the AE parameters by the symmetric ring current, the index of which is SymH.

The MLat position of the AE in the daytime sector correlates with three other indices: $\mathrm{AsyH}, \mathrm{AL}$, and IndN. The $\mathrm{AE}$ shifts with increasing disturbances toward lower latitudes, namely the WE from 72 to $66^{\circ}$ and the EE from 70 to $57^{\circ}$ (Fig. 4b-d). The largest correlations of MLat are found with the IndN coupling function (WE, $r=-0.74$ ), with the smallest values for AsyH (EE, $r=-0.49$ ). IndN is proportional to the amount of opened magnetic flux per unit time in the polar cap, which in turn affects the current systems.

The AL and AsyH indices characterize the large-scale current systems, the magnetic fields of which influence the magnetic field configuration of the dayside sector. Correlations with the ground-based AL index are similar to the AsyH behaviour (cf. Tables 2 and 3). It should be noted that there 

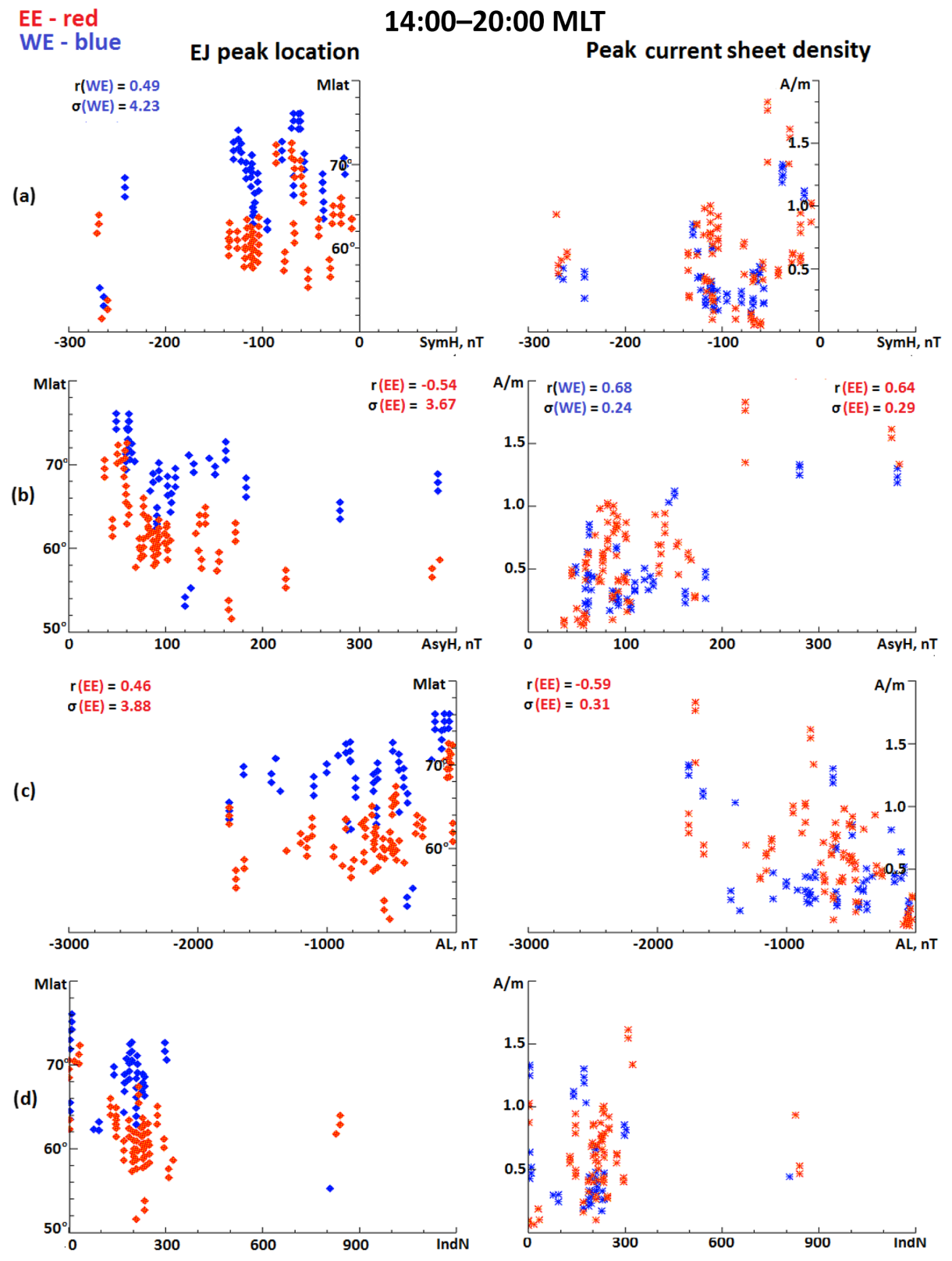

Figure 5. The same as in Fig. 4, but for the evening sector (14:00-21:00 MLT).

are tendencies for the WE to be located a few degrees more poleward than the EE during daytime hours. These tendencies are clearly visible with regard to the MLat(EE and WE) positions and their relation to AsyH and IndN (Fig. 4b and d). The constant term $A$ is larger for the WE than for the EE in the case of AsyH $3.8^{\circ}$ and is $1.9^{\circ}$ larger in the case of the solar wind coupling function IndN.

The correlation coefficients for the Hall current with the IMF $B_{y}$ vector component and its magnitude is low (not shown). A significant correlation coefficient $|r|>0.49$ is achieved in the daytime sector only for the MLat positions of the electrojets, while the correlation with the current den- sities is minimal for all indices. The electrojets can be both westward and eastward. The EE can be observed for very intense disturbances during the storm period down to MLat $57^{\circ}$.

\subsubsection{Evening sector 14:00-21:00 MLT}

Figure 5 provides data presentations in the same format as Fig. 4, but now for the evening sector. Significant correlation values $r$ exist in the evening sector for both the current densities and the MLat positions of the electrojets. The largest values of $r \sim 0.6-0.7$ were obtained for current densities $I$, 


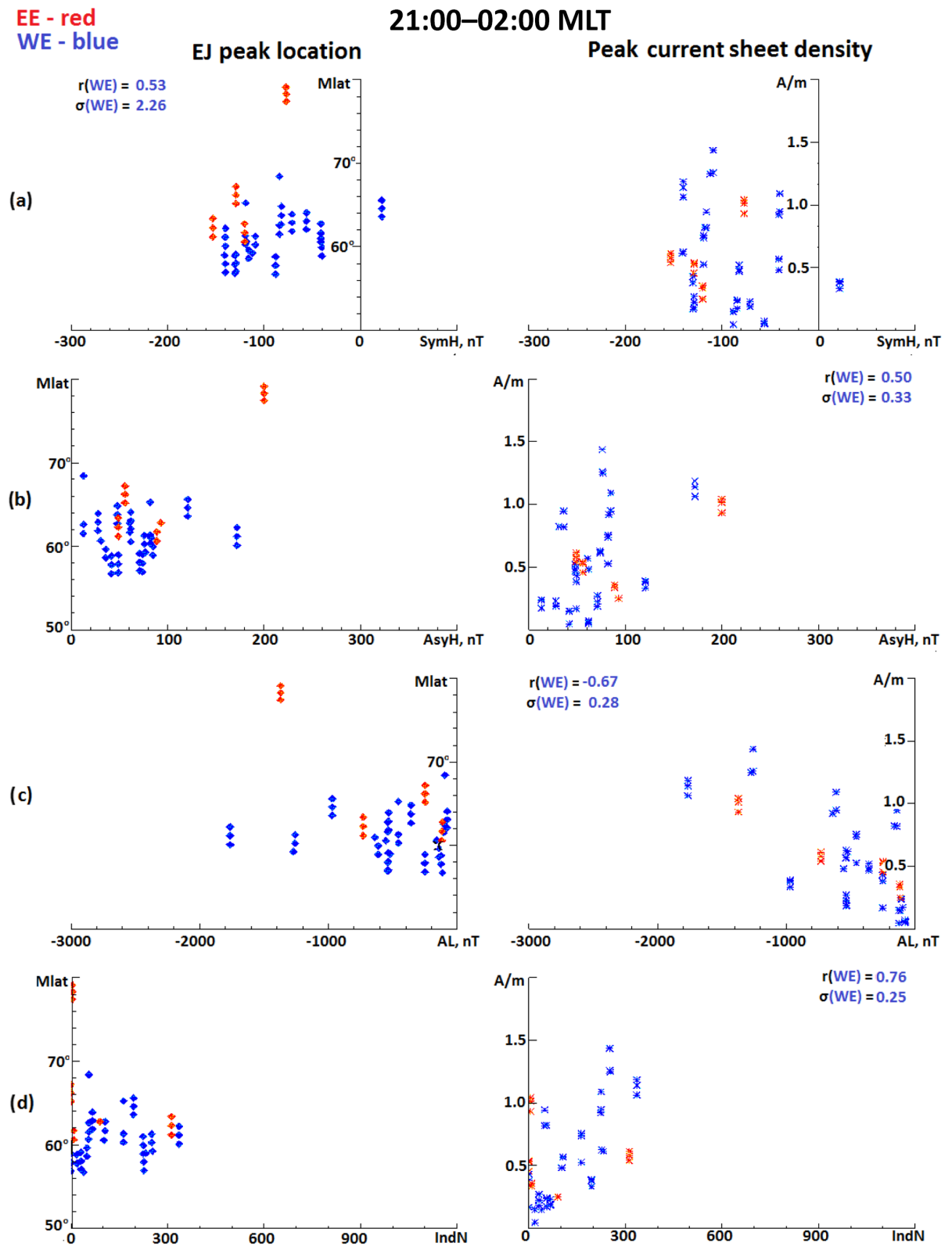

Figure 6. The same as in Fig. 4, but for the midnight sector (21:00-02:00 MLT).

independent of the current directions (westward or eastward) in the electrojets.

A dependence of MLat(WE) appears from the SymH index; the electrojets shifts equatorward with an increase in the ring current. The EE is located more equatorward than the WE by about $\sim 6^{\circ}$. The constant term $A$ of the regression equations amounts accordingly to $\operatorname{MLat}(\mathrm{EE}) \sim 66^{\circ}$ with respect to AsyH and MLat(WE) $\sim 72^{\circ}$ with respect to SymH.

The EE current strength exceeds that of the WE. This means that the interpretation of the EE in the evening sector as branching off from the WE at higher latitudes will become more unlikely (Feldstein et al., 2006).
The electrojets move more equatorward with increasing disturbance levels, according to any geomagnetic activity index. Their current densities rise from $<0.2$ to $1.6 \mathrm{~A} \mathrm{~m}^{-1}$ for the EE and up to $1.3 \mathrm{~A} \mathrm{~m}^{-1}$ for the WE. The current strength of the $\mathrm{EE}$ increases; hence, it is stronger than that of the WE (by about $30 \%$ ). The EE is observed equatorward of MLat $\sim 60^{\circ}$ during magnetic storm periods with the threshold latitude for the EE shift of $\sim 53^{\circ}$. 


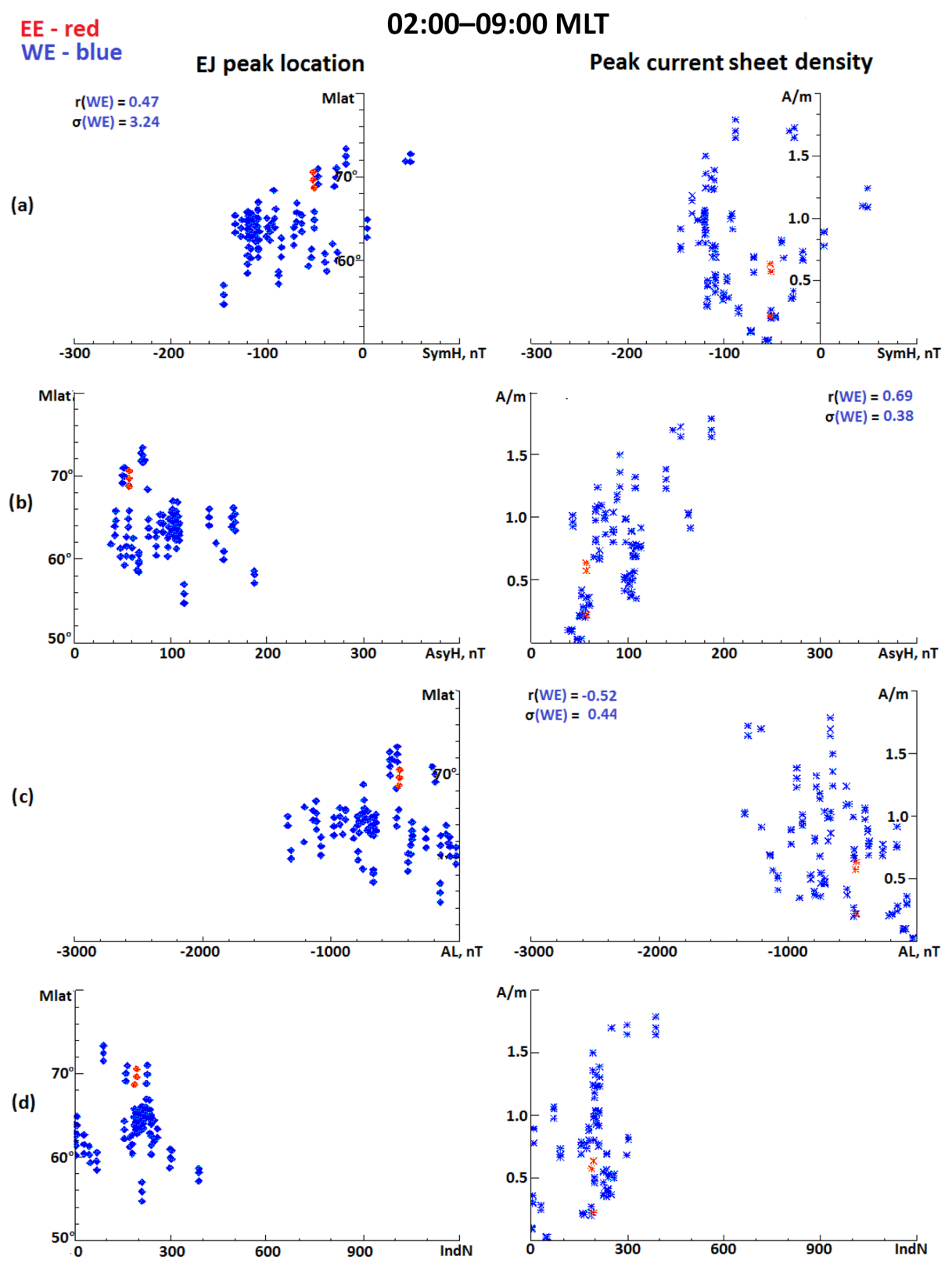

Figure 7. The same as in Fig. 4, but for the morning sector (02:00-09:00 MLT).

\subsubsection{Midnight sector 21:00-02:00 MLT}

In this sector, the WE exists almost exclusively (Fig. 6). Moreover, the current intensity correlates well here with the AsyH, AL, and IndN indices, with a maximum value of $r=0.76$ for the IndN coupling function.

The MLat(WE) position, however, correlates only with the SymH index. It decreases from 62 to $58^{\circ}$ for a change in SymH from $\sim-40$ to $\sim-170 \mathrm{nT}$. The lowest possible MLat appears to be $\sim 58^{\circ}$.

The WE current strength increases from values $<0.2$ to $\sim 1.5 \mathrm{~A} \mathrm{~m}^{-1}$ for an intensification of the disturbance accord- ing to the IndN coupling function from 0 to $325 \mathrm{MWb} \mathrm{s}^{-1}$ (megawebers per second), while the WE position may have an equatorward threshold value of $\sim 58^{\circ}$.

\subsubsection{Morning sector 02:00-09:00 MLT}

Similar to the midnight sector, the WE exists also almost exclusively within the morning sector (Fig. 7). The current intensity correlates well here with the AsyH and AL, with a maximum value of $r=0.69$ for the AsyH index. The WE current strength increases from values 0.32 to $1.92 \mathrm{~A} \mathrm{~m}^{-1}$, with an increase in the AsyH value from 40 to $200 \mathrm{nT}$, while 
the electrojet position moves equatorward until a threshold value of $\sim 56^{\circ}$.

The MLat (WE) position correlates only with the SymH index. In this regard the midnight and morning sectors show the same behaviour. The MLat positions are controlled predominantly by the SymH index, i.e. by the intensity of the ring current DR rather than by any other current system.

The central plasma sheet of the magnetospheric tail is the source region of the WE in the nighttime sector. An increase in the DR is accompanied by a change in the geometry of the magnetic field lines that are interconnected with the central plasma sheet. This results in a shift of the ionospheric projection of the WE toward the equator. The AL index likewise characterizes the westward electrojet WE, but the method of its derivation differs. The AL index indicates the maximum decrease in the horizontal magnetic component of a longitudinal chain of observatories, which is equivalent to the current intensity in westward direction for a given UT moment. The WE, on the other hand, marks the maximum current intensity in westward direction over the meridional sector and the time interval of the spacecraft's orbit. The differences in the methodology of their determination results in rather low correlation values and in the MLT dependent variations of the correlation.

\section{Conclusions}

In this paper we investigated the strength and spatialtemporal distribution (versus magnetic latitude MLat and MLT) of Hall currents at high latitudes. The currents were determined from measurements of scalar magnetic field data, sampled on board the CHAMP satellite at ionospheric altitudes of $\sim 430 \mathrm{~km}$ (Ritter et al., 2004a) during a selection of six magnetic storms (see Table 1). The main findings obtained are as follows.

The current intensity of the PE increases with the magnitude of the IMF $B_{y}$ component, while no correlation at all could be found between the MLat position of the PE and the IMF $B_{y}$ component. The PE is directed eastward for IMF $B_{y}>0$ and westward for IMF $B_{y}<0$. Changes of current flow direction in the PE can occur manifold during the storm, but only due to changes in the IMF $B_{y}$ orientation. There is a strong correlation between the PE current strength and the extent of the ring current asymmetry as indicated by the AsyH index, while there is no connection with the $\mathrm{SymH}$ index, the symmetric part of the ring current. There is an IMF $B_{y}$ control of the magnetic field asymmetry inside the magnetosphere that manifests in the high correlation between AsyH and the PE current intensity.
Auroral electrojets are located at auroral latitudes (MLat $<$ $72^{\circ}$ during daytime hours and MLat $<68^{\circ}$ during nighttime) and exist during every MLT. The number of electrojets in a certain latitude range, the structure of the currents in them, and the interconnection with concrete magnetospheric plasma domains depend on the disturbance level, which is controlled by UT as well as local time (MLT) at the observational points. Around midnight, the WE is predominant, and it exists almost exclusively in the morning sector. During daytime hours, the MLat positions of the auroral electrojets, both WE and EE, correlate with the activity indices AsyH, $\mathrm{AL}$, and IndN. The correlation with the current intensities, however, is relatively small for all indices. The largest correlations $(r \sim 0.6-0.7)$ exist between the AsyH index and the current intensity of the electrojets in the evening sector.

Certain characteristic features of the electrojets appear during the different phases of a geomagnetic storm. With the development of the main phase, both the daytime EE and the nighttime WE shift to sub-auroral latitudes of MLat $\sim 56^{\circ}$, while they increase in strength up to $I \sim 1.5 \mathrm{~A} \mathrm{~m}^{-1}$. During evening hours, the WE is located $\sim 6^{\circ}$ closer to the pole than the EE. A splitting of the WE is possible in the morning hours during the recovery phase, analogous to the splitting of auroral luminescence in the auroral oval.

During evening and nighttime hours the EE is located in the region of diffuse aurora, equatorward of the discrete auroral forms, and projects along magnetic field lines into the inner magnetosphere between the plasmasphere and the central plasma sheet of the magnetospheric tail (Galperin and Feldstein, 1996). In addition to this basic term, an appreciable contribution to the $\mathrm{EE}$ comes during daytime hours from the PRC, which is situated in the near-noon sector of the equatorial magnetosphere near the magnetopause. The WE comprises nighttime MLT from the morning to evening hours and is located in the central plasma sheet, projecting along the magnetic field lines into the auroral oval. The generation of the WE takes place deep inside the plasma sheet, far from magnetic field lines that form the polar cap boundary on the nightside. The PE is controlled by the IMF $B_{y}$ component and is closely related to dayside reconnection processes, which cause the increase in open magnetic flux in the polar cap.

Data availability. The data used in this study are available at http: //doi.org/10.5880/GFZ.2.3.2018.007 (Ritter, 2018). 
Appendix A: Detailed description of the dynamics of further storm intervals

\section{A1 The magnetic storm on 24 August 2005}

This storm began with a sudden storm commencement (SSC) at 06:15 UT, which appeared as a jump-like increase in the SymH index up to $\sim 30 \mathrm{nT}$. The storm phases were identified according to the 1 min values of the SymH index. Figure A1 shows the magnetic activity indices SymH, AsyH, AU, AL, and the IMF components $B_{y}$ and $B_{z}$.

The orbits 29012 and 29013 take place during the growth phase of the storm, the orbits 29014 and 29015 during the main phase, and the orbits 29016-29020 during the recovery phase. The direction and strength of the Hall currents along the orbits are shown in Fig. A2 during daytime hours on the left-hand side, corresponding to the ascending orbital sections, and on the right-hand side during nighttime hours for descending orbital sections. The crossings of the auroral oval occur between 12:00-13:00 MLT during daytime and 23:00-24:00 MLT for the nighttime column. Positive values denote an eastward current (EE) for the descending orbits and a westward current (WE) for the ascending orbital sections.

The index values during the growth phase of the storm are in the range of 25.5-32.7 nT for SymH and 121.1-72.0 nT for AsyH, while the substorms achieve $\sim-1000 \mathrm{nT}$ according to the AL index. SymH intensifies during the main phase up to $-155.6 \mathrm{nT}$, and AsyH intensifies to $206.6 \mathrm{nT}$, where intense substorms with $\mathrm{AL} \sim-3000 \mathrm{nT}$ occur. AsyH values decrease to $43.5 \mathrm{nT}$ during the recovery phase, and we observe weakly variable SymH index values around $-120 \mathrm{nT}$ (see Fig. A1).

An EE exists during daytime hours of orbit 29012, with a current intensity of up to $1.37 \mathrm{~A} \mathrm{~m}^{-1}$ at MLat $72.6^{\circ}$. During the subsequent orbit, the eastward current intensity diminishes to $0.44 \mathrm{~A} \mathrm{~m}^{-1}$ at MLat $70.3^{\circ}$.

The intensification of SymH during the main storm phase (orbit 29015) is accompanied by a continuing decrease in the eastward Hall current to $0.38 \mathrm{~A} \mathrm{~m}^{-1}$ at MLat $=65.4^{\circ}$. An EE with a strength of $\sim 0.7 \mathrm{~A} \mathrm{~m}^{-1}$ is recorded around midday at Mlat $=57.3^{\circ}$, i.e. below $60^{\circ}$, only in connection with very intense substorms (Fig. A1, orbit 29014). Eastward currents at such low latitudes are missing during the other orbits of this storm period. The variations of the AsyH index reflect the variations of the Hall current intensity quite clearly; it attenuates from the orbits 29012 to 29013 and increases during orbit 29014, while it decreases again during orbit 29015.

A westward current in the daytime occurs at MLat $72^{\circ}-$ $80^{\circ}$, beginning with orbit 29015 and continuing until orbit 29020 , i.e. throughout the recovery phase and in the absence of intense substorms. The currents achieve a maximum strength of $I=1.53 \mathrm{~A} \mathrm{~m}^{-1}$ during orbit 29018 at MLat $76.3^{\circ}$. This current is controlled by IMF $B_{y}>0$ and changes its direction with the IMF $B_{y}$ orientation. It is therefore definitively a PE.

The currents in the midnight sector (Fig. A2, right column) are generally directed westward with weak intensity. The only exception occurs during orbit 29014, where the current intensity achieves $I \sim 1.2 \mathrm{~A} \mathrm{~m}^{-1}$. This orbit coincides with the development of a very intense substorm, where the Hall current distribution is very broad, with two maxima of the current intensity at MLat 61.2 and $73.0^{\circ}$. Such a broad latitudinal distribution of the auroral luminescence, with various maxima at different latitudes, is characteristic of the recovery phase of an auroral substorm (Elphinstone et al., 1996). But for the present storm on 24 August 2005, the broad splitup in latitude appeared in the Hall currents during the main phase of the storm.

Summarizing the results of Hall current observations by the CHAMP satellite during the magnetic disturbance period of 24 August 2005 in the daytime and nighttime sectors (11:00-13:00 MLT and 23:00-24:00 MLT, respectively), we conclude the following for the midday sector:

- An EE with a current strength of $1.37 \mathrm{~A} \mathrm{~m}^{-1}$ exists during the growth phase at MLat $\sim 73^{\circ}$ for substorms in the auroral zone with intensities of $\mathrm{AL} \sim-1000 \mathrm{nT}$.

- The EE is observed at MLat $<60^{\circ}$ during the main storm phase for intense substorms with intensities of $\mathrm{AL} \sim-3000 \mathrm{nT}$.

- The variations of the EE intensities during the growth and main phases of the storm occur synchronously with the AsyH index. Comparable variations with the $\mathrm{SymH}$ index are not observed.

- Currents directed westward or eastward are observed during the recovery phase at $72^{\circ}<$ MLAT $<80^{\circ}$ with a maximum intensity of $\sim 0.9 \mathrm{~A} \mathrm{~m}^{-1}$. Their direction is controlled by the IMF $B_{y}$ component, i.e. they are in accordance with the PE.

In addition, we conclude the following for the midnight sector:

- As a rule, the Hall currents are directed westward during nighttime. In the concrete observations, the WE can be split into several parts with several maxima versus latitude.

\section{A2 The magnetic storm on 18 June 2003}

Figure A3 shows the variations of the SymH and AsyH indices for the magnetic storm on 18 June 2003. The storm phases are represented by the orbit numbers 16532 and 16533 for the growth phase, 16534-16536 for the main phase, and 16537-16541 for the recovery phase. Extreme values of SymH and AsyH are observed during the main 
24 Aug 2005

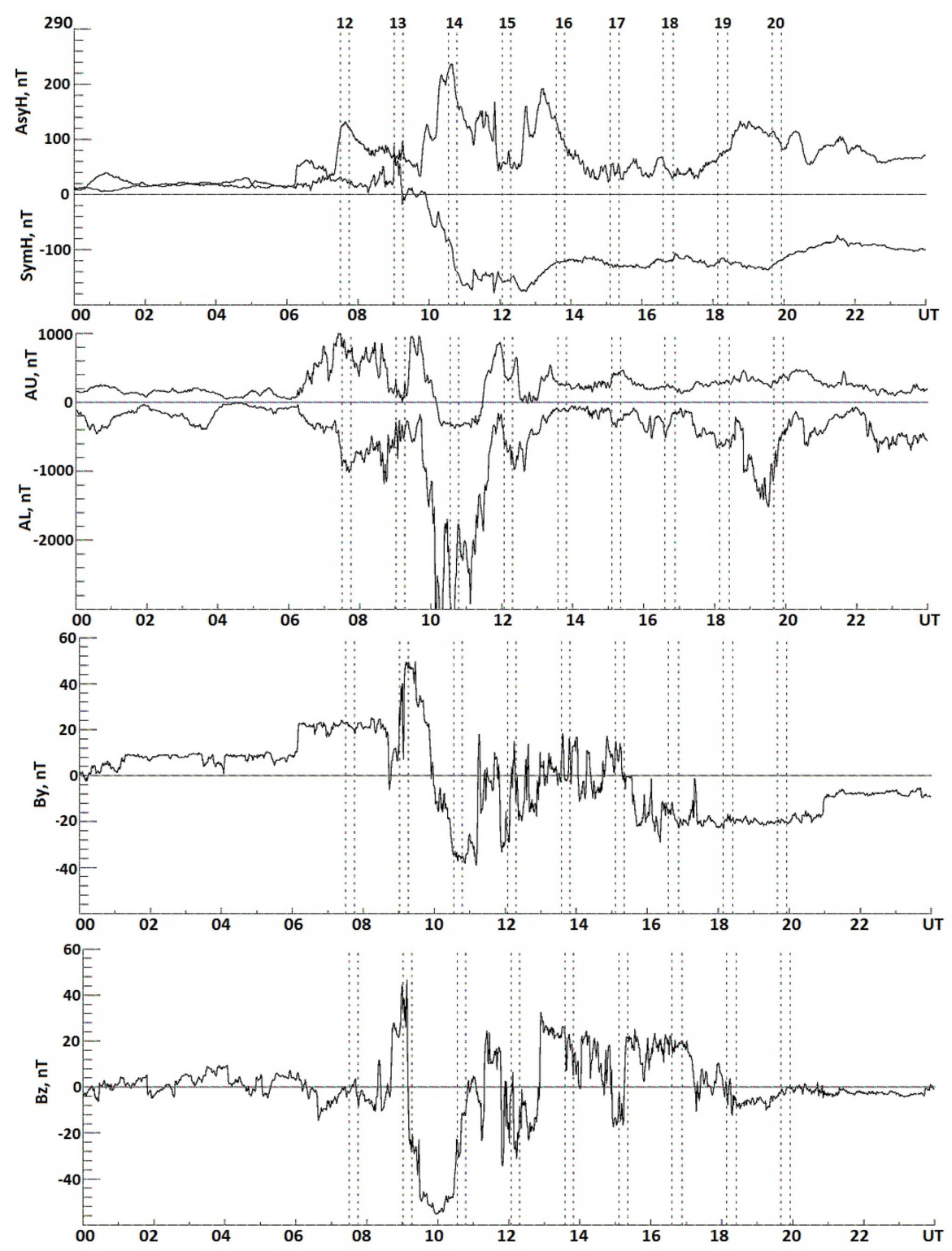

Figure A1. One-minute values of the AsyH, SymH, AU, and AL indices and of the $B_{y}$ and $B_{z}$ components of the IMF for the storm on 24 August 2005 (analysis interval from 07:00-20:00 UT, orbits 29012-29020). The time of each orbit and its orbit number are indicated as in Fig. 1 of the paper.

phase with -163 and $91 \mathrm{nT}$, respectively, while the substorm index AL achieves $-1298 \mathrm{nT}$. The CHAMP trajectories are situated during this storm period along the meridional plane of 13:00-14:00 MLT (afternoon) and 00:0002:00 MLT (near midnight). In the daytime sector, a EE exists during the growth phase at MLat $\sim 67^{\circ}$ with $I \sim$ $0.43 \mathrm{~A} \mathrm{~m}^{-1}$, and a WE exists at MLat $\sim 72^{\circ}$ with $I \sim$ $0.42 \mathrm{~A} \mathrm{~m}^{-1}$. Both electrojets are retained during the main storm phase with an EE of $I \sim 0.8 \mathrm{~A} \mathrm{~m}^{-1}$ at MLat $\sim 62^{\circ}$ and a WE of $I \sim 0.5 \mathrm{~A} \mathrm{~m}^{-1}$ at MLat $\sim 67^{\circ}$. The WE only per- sists during the recovery phase with $I \sim 0.3 \mathrm{~A} \mathrm{~m}^{-1}$ at MLat $\sim 78^{\circ}$ (orbits 16537 and 16538) This high-latitude westward current near MLat $\sim 77^{\circ}$ with $I \sim 0.4 \mathrm{~A} \mathrm{~m}^{-1}$ does not vanish till the end of the recovery phase. Such a high-latitude position of a westward current near-noon MLT gives reason to suggest that this is a polar electrojet (PE). This assumption would apply if the IMF $B_{y}$ component is negative. Indeed, the $B_{y}$ component appeared to be at a steady negative value during the orbits 16537-16541. 
(a)

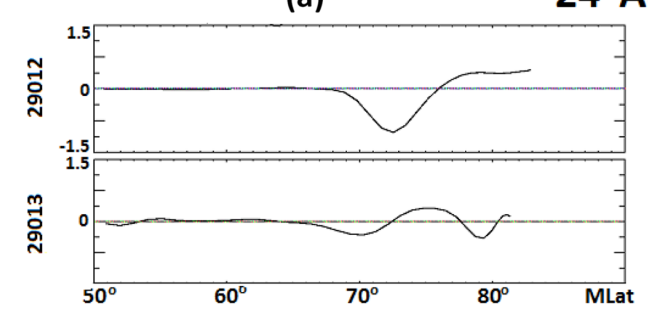

Main phase

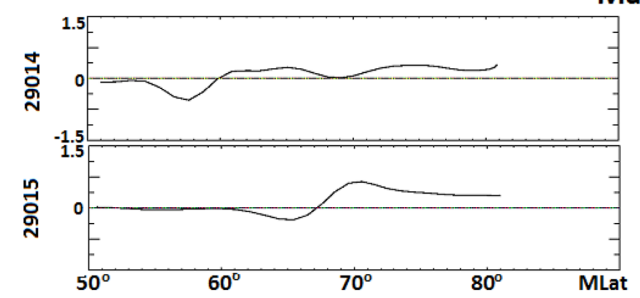

Recovery phase

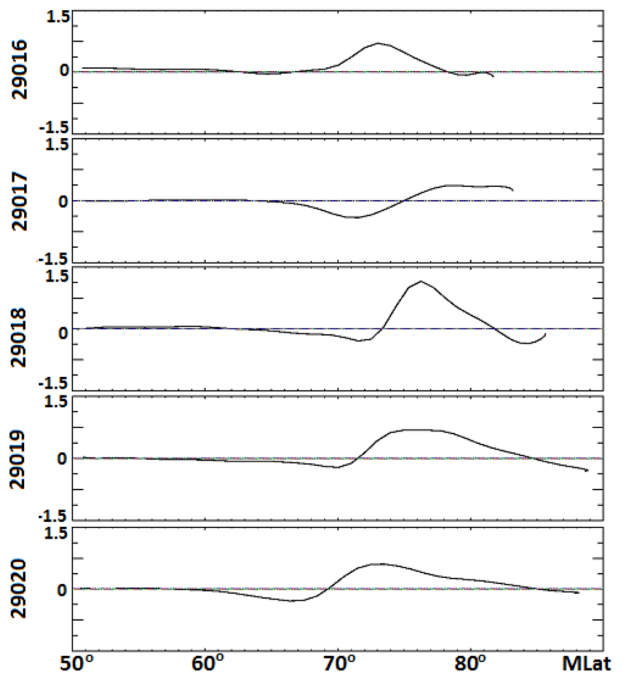

(b)
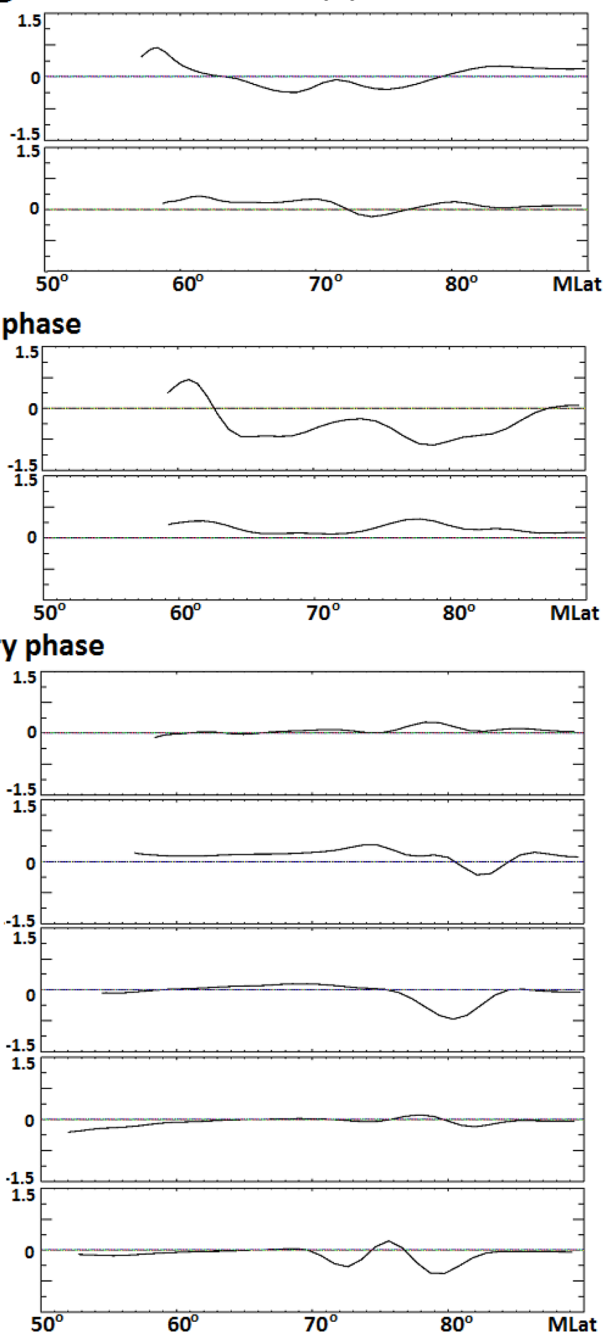

Figure A2. Direction and intensity values of the Hall current along the satellite orbit at the dayside (a, 11:00-13:00 MLT, corresponding to the ascending section of the orbit) and nightside sectors (b, 23:00-24:00 MLT, descending orbit section). Positive currents denote an eastward current for the descending orbit section and a westward current for the ascending section, respectively.

As a rule, the ionospheric currents in the nighttime sector are directed westward in the MLat range of 57.8-63.0 with $I \sim 0.5 \mathrm{~A} \mathrm{~m}^{-1}$. Only during two orbits in the growth and main phases, the current strength achieved is represented by $I \sim(1.1-1.4) \mathrm{A} \mathrm{m}^{-1}$.

It should be noted that this storm had relatively intense SymH values, while the AsyH values remained, however, at a relatively low level. The EE and WE intensities were small as well.

Summarizing the results of Hall current observations by the CHAMP satellite during the magnetic disturbance period of 18 June 2003 in the daytime and nighttime sectors (13:00-14:00 MLT and 00:00-02:00 MLT, respectively), we conclude:
- The quite strong geomagnetic storm (according to the $\mathrm{SymH}<-150 \mathrm{nT}$ index value during the main phase) is accompanied by substorms with AL up to $-1500 \mathrm{nT}$ and with the uppermost index value for the asymmetry of the field AsyH $<100 \mathrm{nT}$. The peculiarities of this storm period obviously caused the appearance of an EE in the daytime sector and a WE in the nighttime sector at MLat $<60^{\circ}$.

- A stable PE with a current intensity up to $0.4 \mathrm{~A} \mathrm{~m}^{-1}$ in the westward direction persists during the recovery phase with an IMF $B_{y}<0 \mathrm{nT}$ component. 


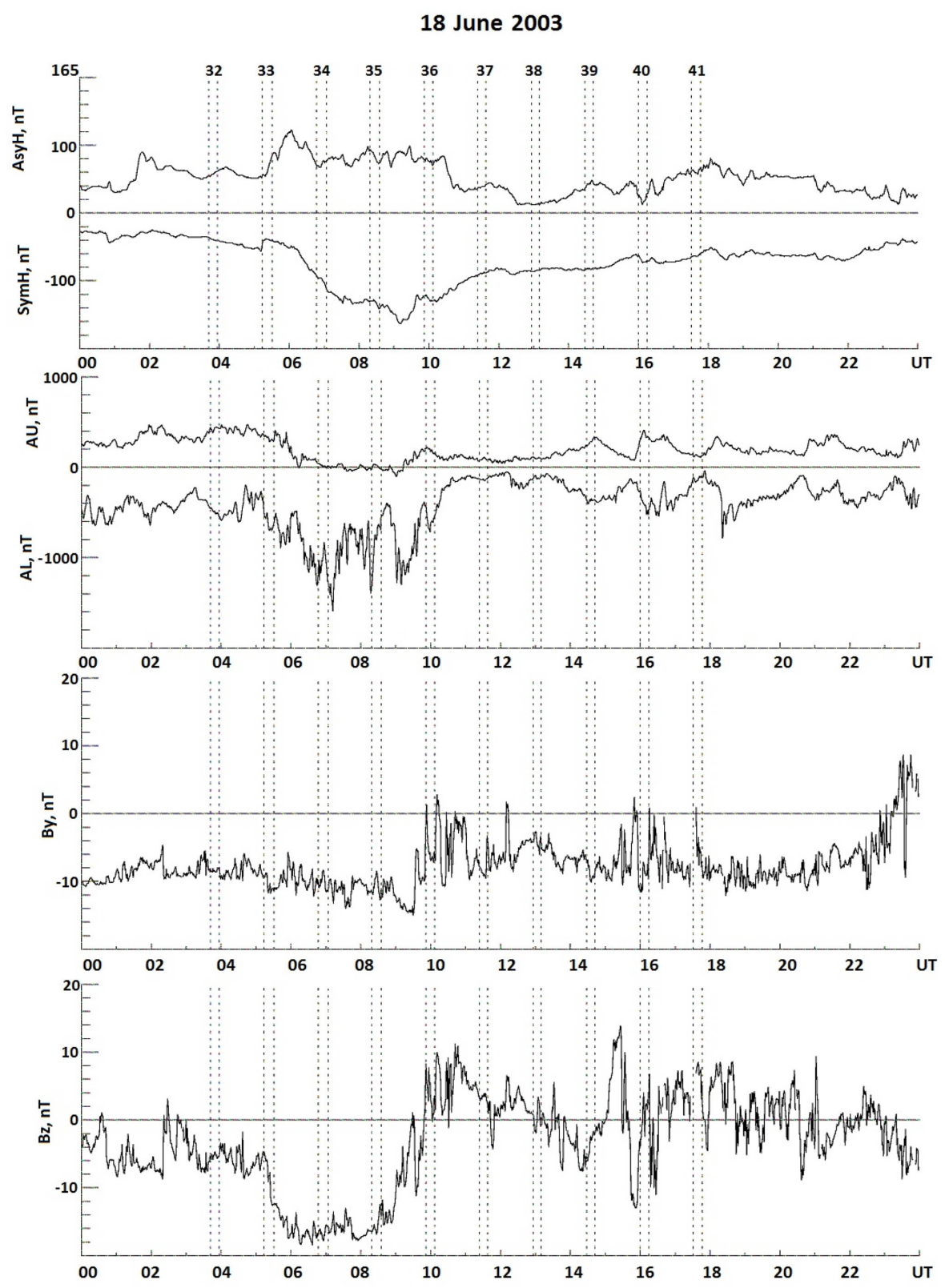

Figure A3. One-minute values of the AsyH, SymH, AU, and AL indices and of the $B_{y}$ and $B_{z}$ components of the IMF for the storm on 18 June 2003 (analyzed interval from 03:00-18:00 UT, orbits 16532-16541). The time of each orbit and its orbit number are indicated as in Fig. 1 of the paper.

\section{A3 The magnetic storm on 30 May 2005}

Figure A5 shows the variations of the SymH, AsyH, AU, and $\mathrm{AL}$ indices for the magnetic storm on 30 May 2005, between 02:00 and 20:00 UT. The vertical dotted lines indicate the time intervals of the satellite crossings over high latitudes of the Northern Hemisphere (MLat $>60^{\circ}$ ), and the numbers denote the satellite's orbit counter. Prior to the storm's onset (orbits 27659 and 27660), the geomagnetic field is relatively quiet according to all indices, including the AL index. It is recovered from -28.6 to $-17.3 \mathrm{nT}$ in terms of $\mathrm{SymH}$, from 37.8 to $17.8 \mathrm{nT}$ for $\mathrm{AsyH}$, and from -40 to $0 \mathrm{nT}$ with respect to the AL index. These changes correspond to a recovery process toward a quiet-time level after the previous disturbance.

The main phase of the magnetic storm starts with a steady increase in SymH from -28.6 nT during orbit 27661, a jumplike increase from 44.5 to $104.2 \mathrm{nT}$ in AsyH during the same overflight, and continues with a decrease in SymH to $-118.4 \mathrm{nT}$ during orbit 27665. The peak values of AsyH and AL during the main storm phase are 162.1 and $-1200 \mathrm{nT}$, re- 
(a)
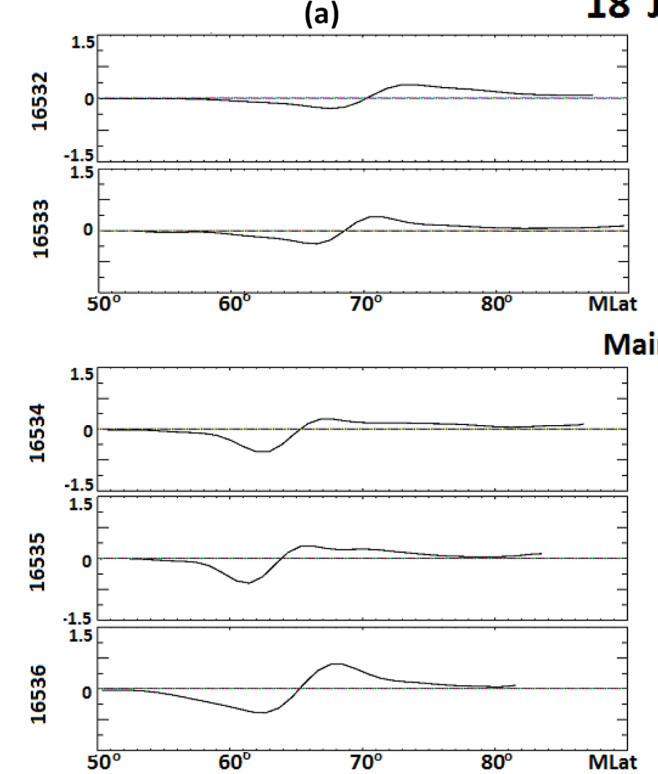

Main phase
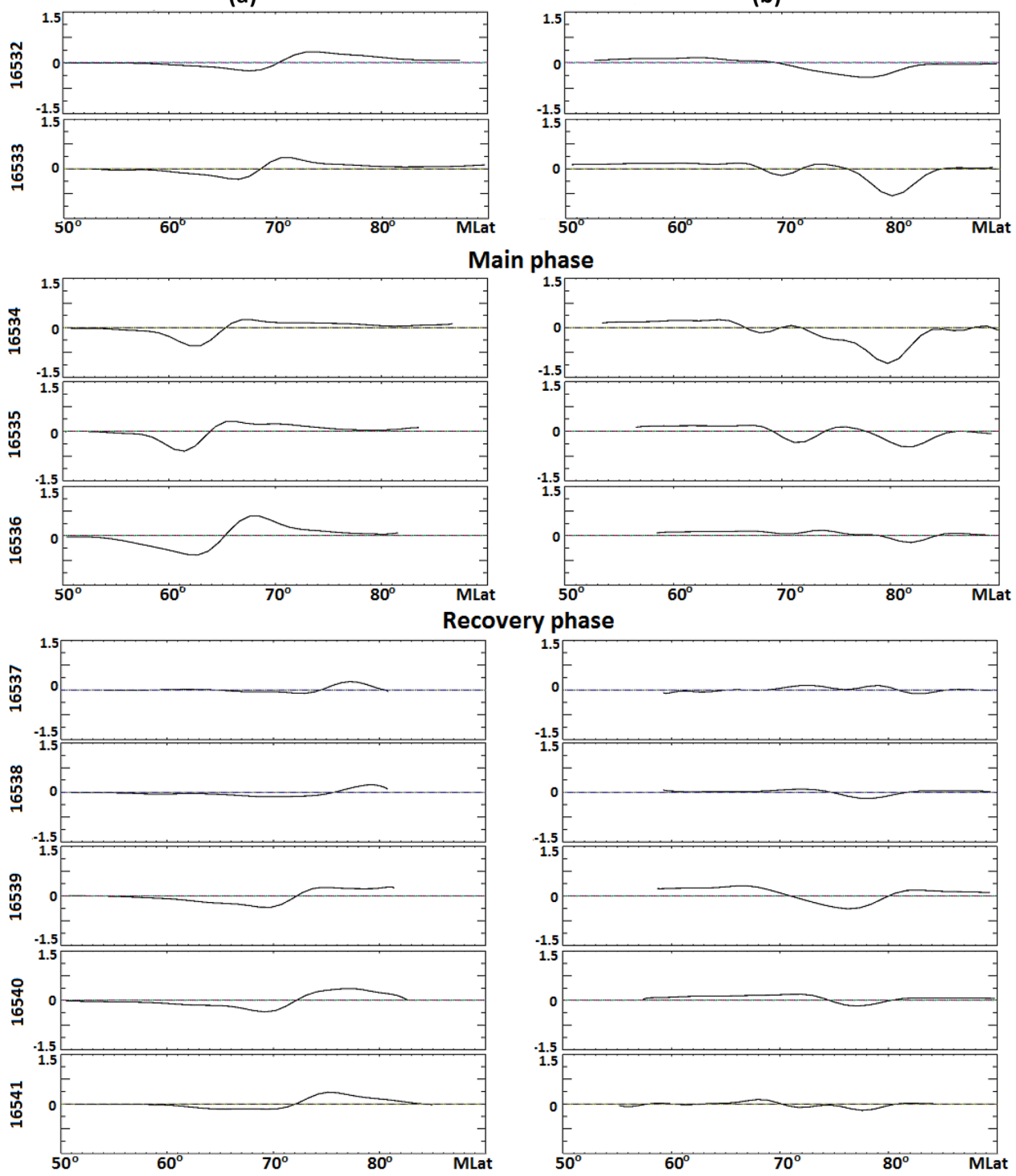

Figure A4. Direction and intensity values of the Hall current along the satellite orbit at the dayside (a, 12:00-16:00 MLT, corresponding to the ascending section of the orbit) and nightside sectors (b, 00:00-04:00 MLT, descending orbit section). Positive currents denote an eastward current for the descending orbit section and a westward current for the ascending section, respectively.

spectively. The recovery phase takes place during the orbits 27666 and 27667, after which, during the orbit 27668, the appearance of a new disturbance is recorded (according to the $\mathrm{AL}$ and SymH indices). The ascending CHAMP trajectory during the storm goes along the 19:00-21:00 MLT meridian (evening), while the descending orbit section is along the 06:00-09:00 MLT meridian in the morning sector. Fig. A6 shows the direction and the strength of the Hall currents for the evening (left side) and morning (right side) sectors.

During the orbits prior to the beginning of the main phase, the Hall current is either missing in the evening sector or exists only in terms of a distributed eastward current with maximum densities of $J \sim 0.3 \mathrm{~A} \mathrm{~m}^{-1}$ at MLat $\sim 66^{\circ}$. In the morning sector, a WE is recorded with $J \sim 0.9 \mathrm{~A} \mathrm{~m}^{-1}$ at MLat $\sim 80^{\circ}$ and MLT $\sim 09: 00 \mathrm{~h}$. The existence of such intense currents during daytime hours at such high latitudes during relatively quiet geomagnetic conditions is unusual. A reasonable explanation might be the assumption that this current concerns the PE. In this case, the orbits investigated should occur during conditions of IMF $B_{y}<0 \mathrm{nT}$. Indeed, according to Fig. A5, a quite stable negative IMF $B_{y} \sim-18 \mathrm{nT}$ is observed prior to the main storm phase. The beginning of the main phase (orbit 27661) is characterized by the appearance of two currents in the evening sector: the $\mathrm{EE}$, with $J \sim 0.6 \mathrm{~A} \mathrm{~m}^{-1}$ at MLat $\sim 63^{\circ}$, and the WE, with $J \sim 0.3 \mathrm{~A} \mathrm{~m}^{-1}$ at MLat $\sim 68^{\circ}$. In the course of the storm, 


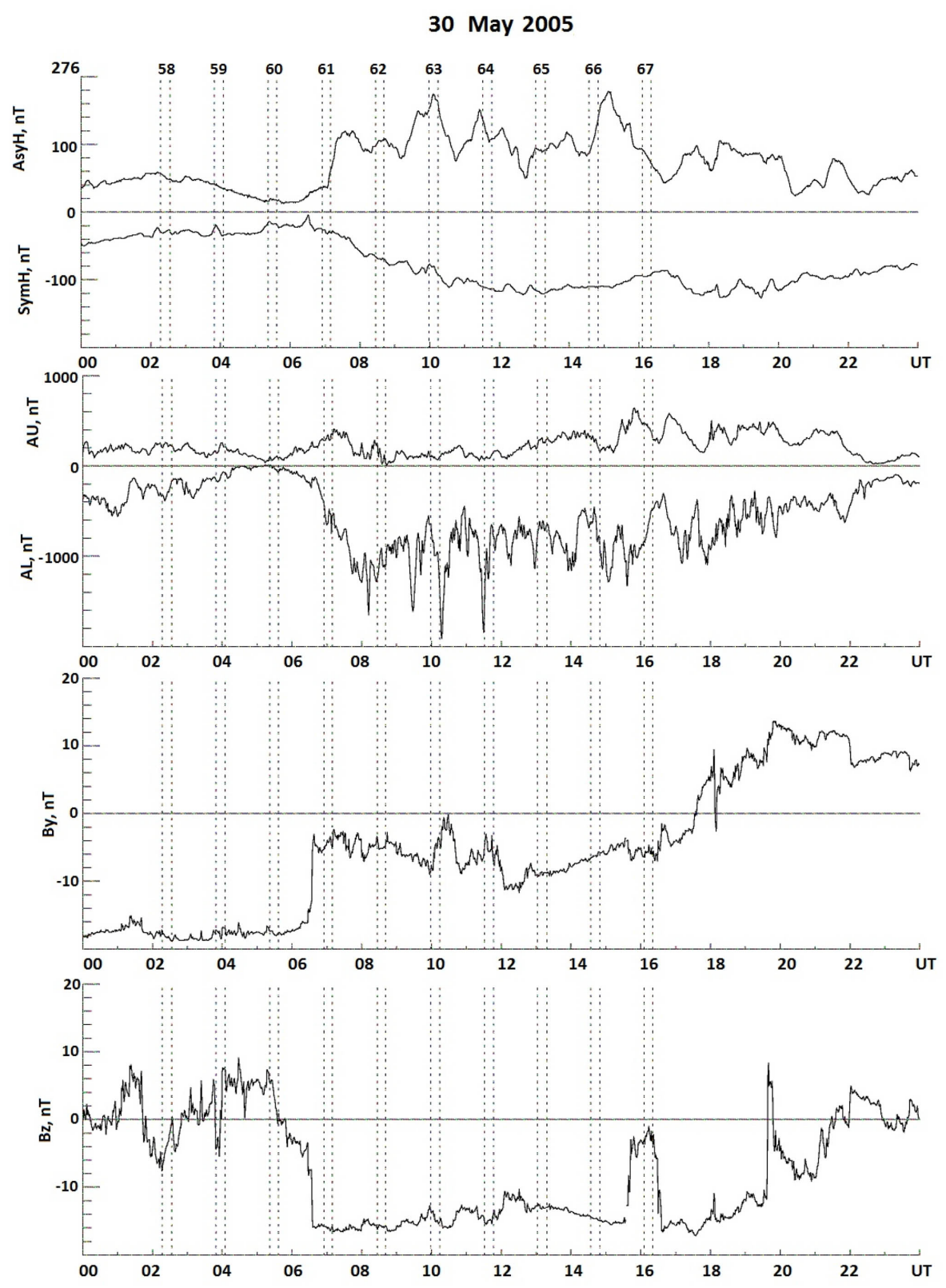

Figure A5. One-minute values of the AsyH, SymH, AU, and AL indices and of the $B_{y}$ and $B_{z}$ components of the IMF for the storm on 30 May 2005 (analysis interval 02:00-17:00 UT, orbits 27658-27667). The time of each orbit and its orbit number are indicated as in Fig. 1 of the paper.

the EE attains a strength of $J \sim 0.7 \mathrm{~A} \mathrm{~m}^{-1}$, shifting equatorward until MLat $\sim 80^{\circ}$. The displacement in MLat toward the equator reflects the more general tendency in which the electrojets move more equatorward with increasing current $J$. The current intensity in the WE remains at $J \sim 0.4 \mathrm{~A} \mathrm{~m}^{-1}$. In the morning sector, the current stays at MLat $\sim 70^{\circ}$, and its current strength during orbit 27661 is kept at $J \sim 0.4 \mathrm{~A} \mathrm{~m}^{-1}$. This is obviously the first appearance of an auroral WE in the morning sector. The WE at auroral latitudes increases during the subsequent orbits and attains $1.5 \mathrm{~A} \mathrm{~m}^{-1}$ during orbit
27665 at MLat $\sim 64^{\circ}$. The recovery phase during orbit 27667 is characterized by a westward current with $J \sim-1.0 \mathrm{~A} \mathrm{~m}^{-1}$ in the morning sector at MLat $\sim 68^{\circ}$ and a weaker current with $J \sim 0.3 \mathrm{~A} \mathrm{~m}^{-1}$ at MLat $\sim 63^{\circ}$. In the course of the storm, the current intensity $J$ in the morning sector exceeds significantly the Hall current intensity values of the same orbit in the evening sector.

Summarizing the results of Hall current observations by the CHAMP satellite during the magnetic disturbance period of 30 May 2005 in the dusk and dawn sectors (19:00- 
(a)

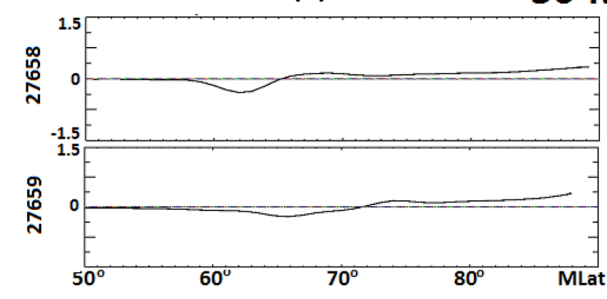

30 May 2005
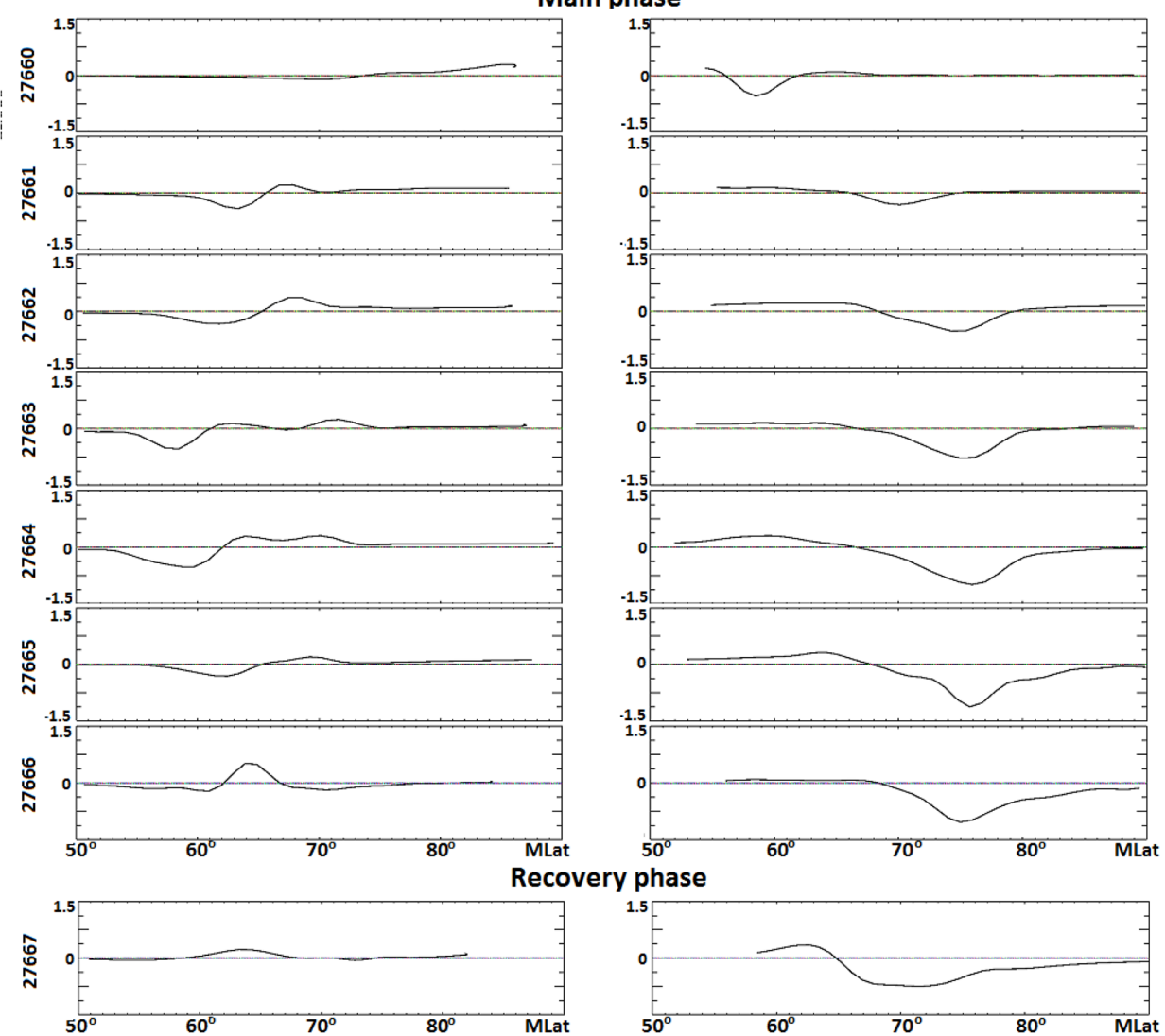

(b)

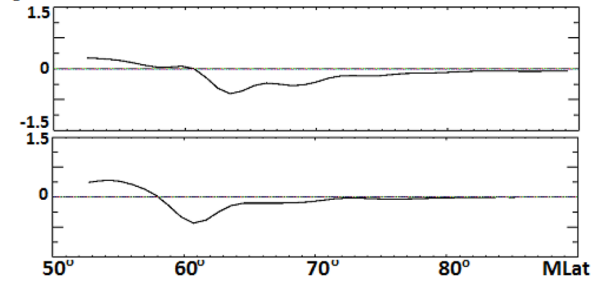

hase

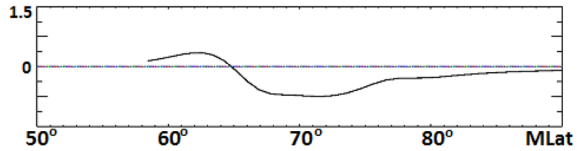

Figure A6. Direction and intensity values of the Hall current along the satellite orbit at the duskside (a, 19:00-21:00 MLT, corresponding to the ascending section of the orbit) and dawnside sectors (b, 06:00-09:00 MLT, descending orbit section). Positive currents denote an eastward current flow for the descending orbit section and a westward current for the ascending section, respectively.

21:00 MLT and 06:00-09:00 MLT, respectively) we conclude the following:

- Two auroral Hall currents (EE and WE) exist in the evening, and only one current (WE) exists in the morning sector.

- The currents are positioned, as a rule, at latitudes MLat of the auroral zone $\left(63-68^{\circ}\right)$. During the main phase, the current can be shifted to MLat $\sim 58.5^{\circ}$.

- In the evening sector, the position of the EE is more equatorward than the WE.

- During early evening hours, the Hall current strength of the EE exceeds the WE current intensity, and in the morning hours, the WE current intensity is larger than during the evening.

- The recovery process toward the quiet-time level can be accompanied at by late evening or polar electrojet (PE) at MLat $\sim 80^{\circ}$ in the late morning hours of the PE with $J \sim 0.8 \mathrm{~A} \mathrm{~m}^{-1}$.

\section{A4 The magnetic storm on 15 May 2005}

Figure A7 shows the variations of the $\mathrm{SymH}, \mathrm{AsyH}$, and IndN indices, as well as the IMF $B_{y}$ and $B_{z}$ components and the solar wind velocity in the interval 00:00-23:00 UT for the magnetic storm on 15 May 2005. The main phase of the magnetic storm takes place during the orbits 27426 and 


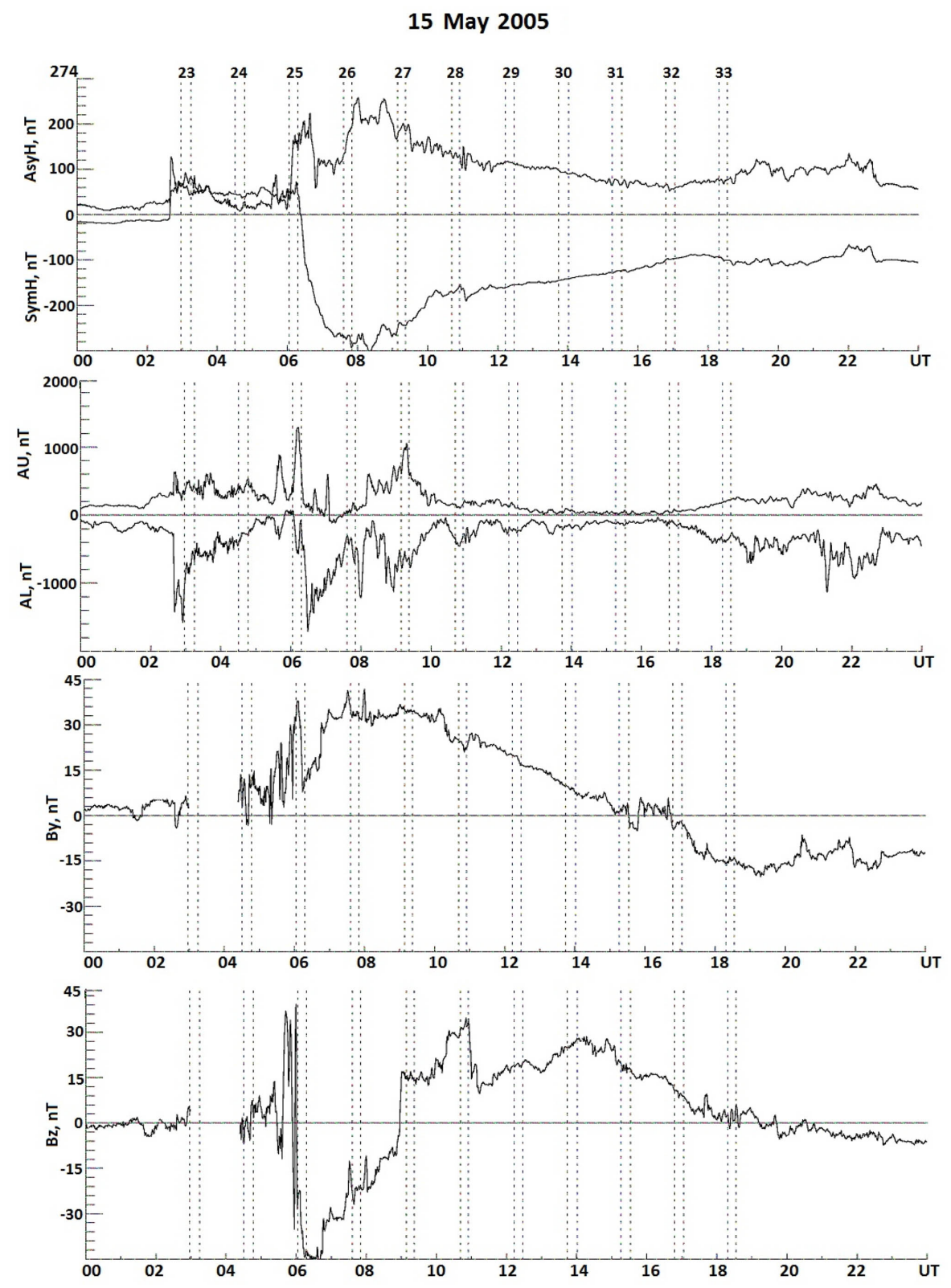

Figure A7. One-minute values of the AsyH, SymH, AU, and AL indices and of the $B_{y}$ and $B_{z}$ components of the IMF for the storm on 15 May 2005 (analysis interval 02:00-19:00 UT, orbits 27423-27433). The time of each orbit and its orbit number are indicated as in Fig. 1 of the paper.

27427 , with a SymH index value of $-274.5 \mathrm{nT}$, an AsyH of $186.1 \mathrm{nT}$, and $\mathrm{AL} \sim-1700 \mathrm{nT}$. The orbits 27423 and 27424 prior to the main phase occur during weakly disturbed magnetic field conditions, with SymH $\sim 50 \mathrm{nT}$ and AsyH $\sim 75$ $16 \mathrm{nT}$. During orbit 27425, with SymH at $48.8 \mathrm{nT}$, the AsyH index increases strongly to $121.3 \mathrm{nT}$, which appears to be the onset of an intense magnetic storm. The recovery phase takes place during the orbits 27428 and 27432, during which a steady decrease in the $\mathrm{SymH}$ index to $-125.2 \mathrm{nT}$ occurs as well as a decrease in the AsyH index value to $70.1 \mathrm{nT}$. The ascending CHAMP trajectory during the storm spread along the 20:00-22:00 MLT meridian, and the descending trajectory is along the 08:00-10:00 MLT meridian in the morning sector. Figure A8 shows the direction and the strength of the Hall currents for the evening (left side) and morning (right side) sectors.

The current strength for the EE during orbit 27425 is, at $\sim 0.4 \mathrm{~A} \mathrm{~m}^{-1}$, quite small in the evening sector prior to the main phase. Both an EE and a WE exist during the main phase, with $J \sim 0.6 \mathrm{~A} \mathrm{~m}^{-1}$. The EE shifts on average to a 
(a)
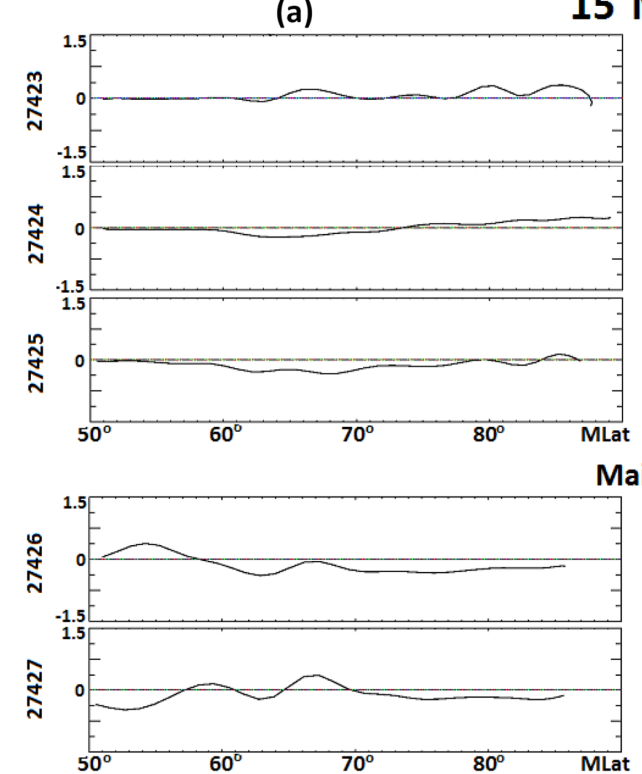

Recovery phase

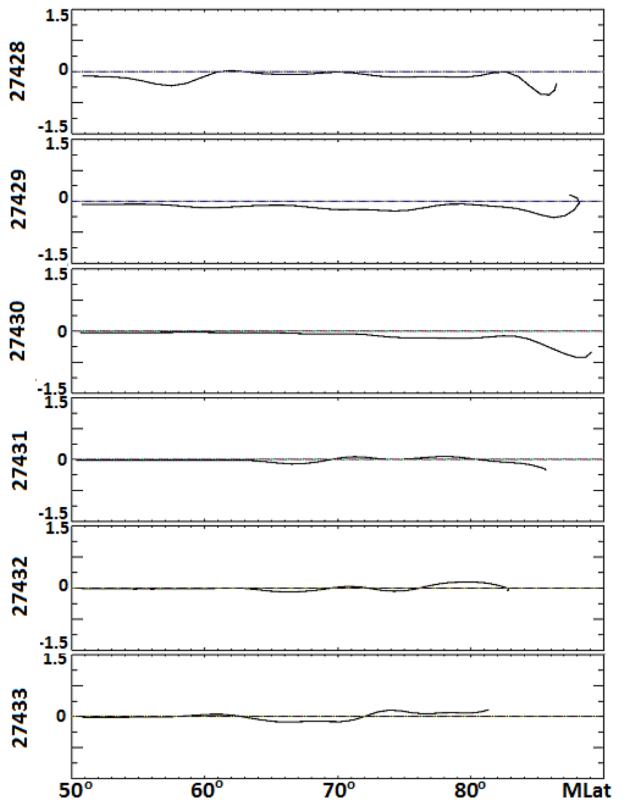

(b)

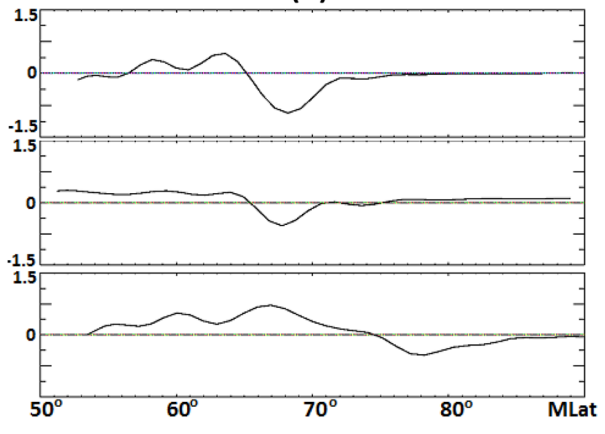

phase
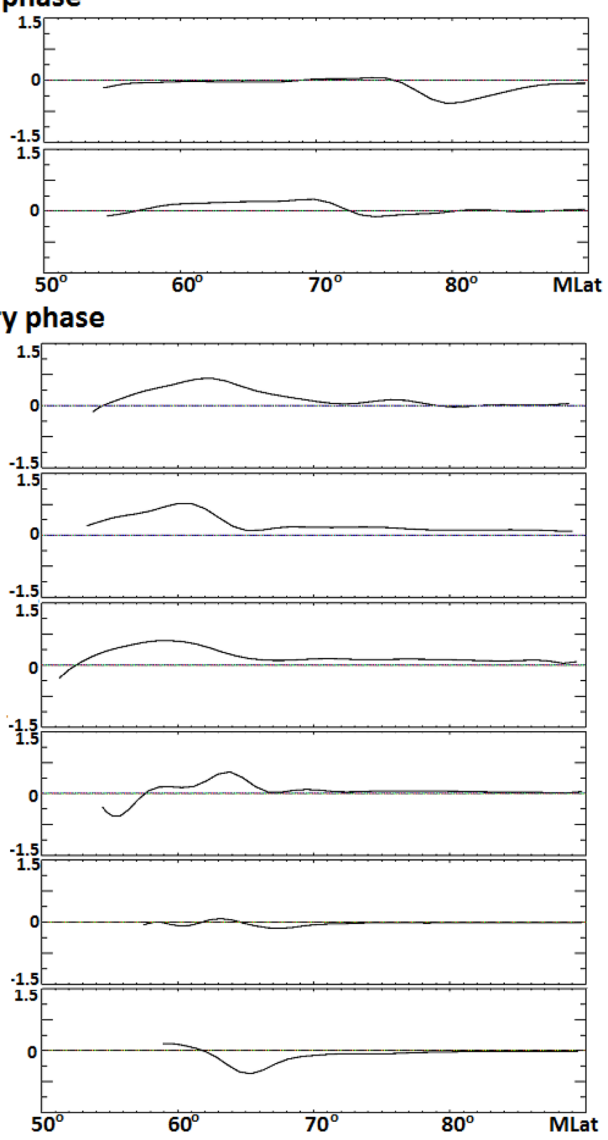

Figure A8. Direction and intensity values of the Hall current along the satellite orbit at the duskside (a, 20:00-22:00 MLT, corresponding to the ascending section of the orbit) and dawnside sectors (b, 08:00-10:00 MLT, descending orbit section). Positive currents denote an eastward current flow for the descending orbit section and a westward current for the ascending section, respectively.

MLat of $\sim 52.5^{\circ}$ with SymH $\sim-250 \mathrm{nT}$. The Hall currents are practically absent during the recovery phase.

In the evening sector, the currents turn out to have difficult characteristics, which change with the storm phases. A WE at MLat $\sim 72^{\circ}$ with $J \sim 1.0 \mathrm{~A} \mathrm{~m}^{-1}$ is recorded during the magnetically quiet period prior to the main phase. With the development of the main phase, the WE shifts to MLat $\sim 61^{\circ}$. During the recovery phase, the WE decays at auroral latitudes, but in the latitudinal range $77^{\circ}<$ Mlat $<80^{\circ}$, an EE appears with $J \sim 1.0 \mathrm{~A} \mathrm{~m}^{-1}$ (orbits 27428-27431). The orbits with an EE coincide temporally with an interval of IMF $B_{y}>0 \mathrm{nT}$ in the solar wind (Fig. A7). All characteristic features of the PE are therefore present here. During orbit 27433, the direction of the current changes to WE. This is accompanied by a corresponding change in the IMF $B_{y}$ orientation, as can be seen in Fig. A7.

The characteristic features of this storm are the following: 
- The quiet-time level of the magnetic field variations prior to the storm main phase can be described as missing or unimportant intensities of the EE and WE Hall currents in the evening sector, while in the morning sector, only the WE at auroral latitudes exists.

- During the main phase of this intense storm with a SymH index of $\sim-250 \mathrm{nT}$ in the evening sector, the WE shifts to MLat $\sim 52.5^{\circ}$, and the WE shifts to MLat $\sim 54.0^{\circ}$.

- A PE appears during the recovery phase in the late morning hours at $77^{\circ}<$ Mlat $<80^{\circ}$, where the Hall currents are controlled by the direction of the IMF $B_{y}$ component.

\section{A5 The magnetic storm on 18 August 2003}

Figure A9 shows the variations of the $\mathrm{SymH}, \mathrm{AsyH}, \mathrm{AU}$, and $\mathrm{AL}$ indices as well as the IMF $B_{y}$ and $B_{z}$ components in the interval 00:00-23:00 UT for the magnetic storm on 18 August 2003. The main phase of the magnetic storm takes place during the orbits $17482-17489$, with peak values of
SymH and AsyH of -133.8 and $101.4 \mathrm{nT}$, respectively, and an AL value of $\sim-1400 \mathrm{nT}$. During the orbits 17480 and 17481 prior to the main phase, the values of SymH and AsyH are -18.4 to -43.3 and $72.0-48.8 \mathrm{nT}$, and during the recovery phase, in the course of orbits 17490-17493, they amount to 115.4 and $56.7 \mathrm{nT}$, respectively. The CHAMP trajectories during the storm spread along the 07:00-09:00 MLT meridian (morning) and along the 19:00-21:00 MLT meridian (evening). Figure A10 shows the direction and the strength of the Hall currents for the morning (left side) and evening (right side) sectors.

The characteristic peculiarities of the spatial-temporal distribution of the FACs during this storm concur with those described for the other storms. During the main phase in the evening sector, as a rule, an EE exists. The EE appears at MLat $\sim 66.5^{\circ}$ with $J \sim 0.6 \mathrm{~A} \mathrm{~m}^{-1}$, and it then shifts equatorward to MLat $\sim 58.8^{\circ}$ with $J \sim 1.0 \mathrm{~A} \mathrm{~m}^{-1}$ during orbit 17486. A WE exists in the morning sector at auroral latitudes of $61^{\circ}<$ Mlat $<65^{\circ}$ with $J \sim 1.2 \mathrm{~A} \mathrm{~m}^{-1}$. A weak eastward current, which is distributed in the polar cap, persists due to the closure of parts of the electrojets across the near-polar region. 

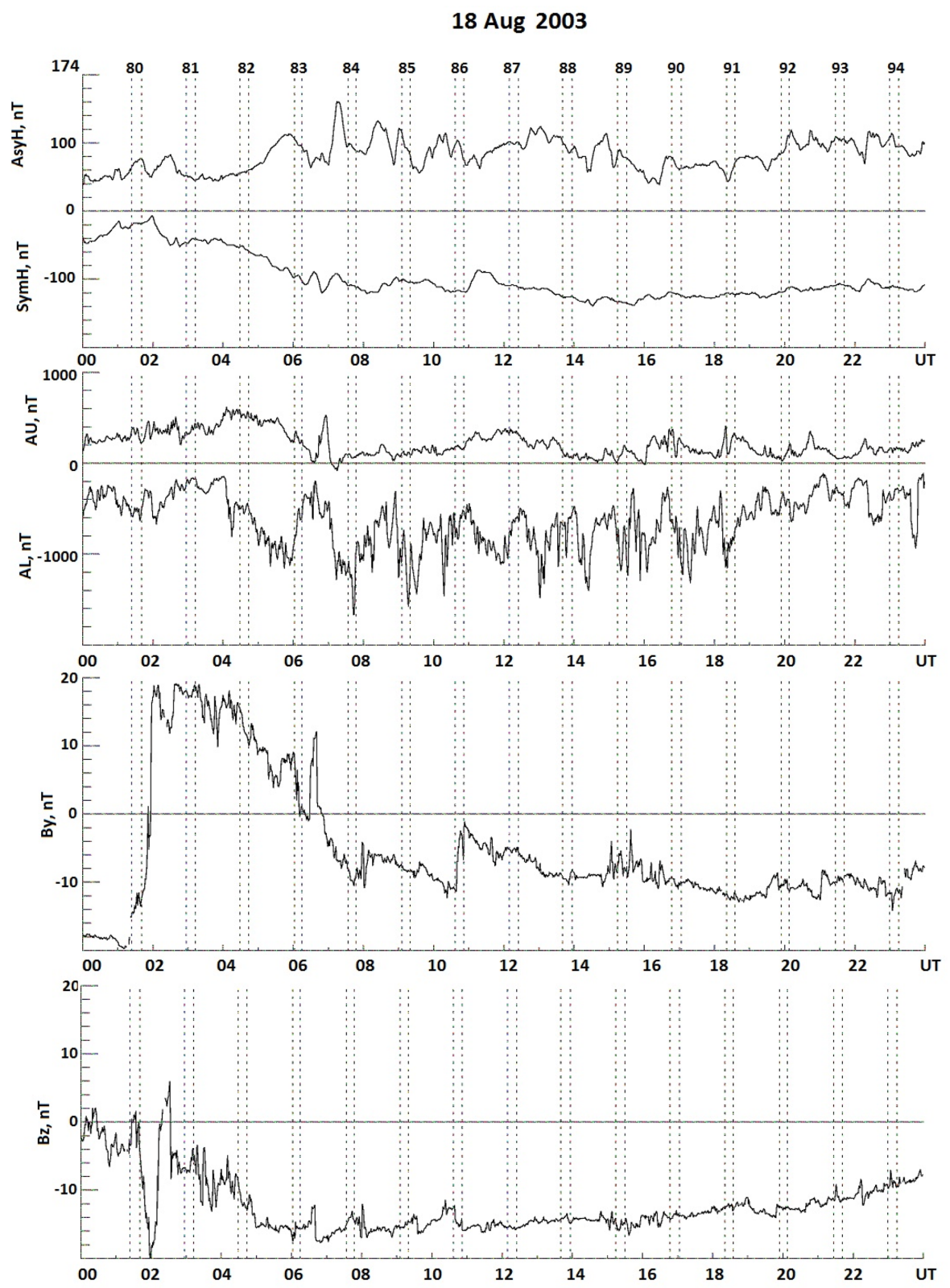

Figure A9. One-minute values of the AsyH, SymH, AU, and AL indices and of the $B_{y}$ and $B_{z}$ components of the IMF for the storm on 18 August 2003 (analysis interval 00:00-23:00 UT, orbits 17480-17494). The time of each orbit and its orbit number are indicated as in Fig. 1 of the paper. 
(a)
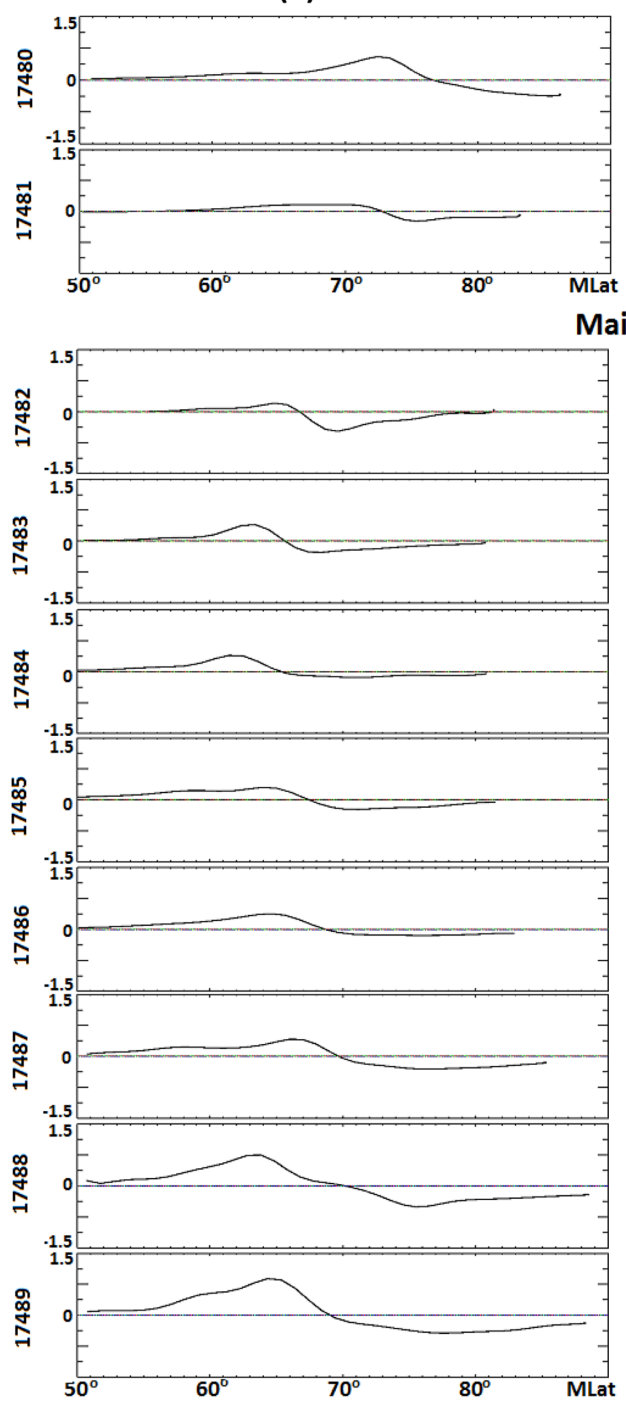

Main phase

18 Aug 2003

(b)
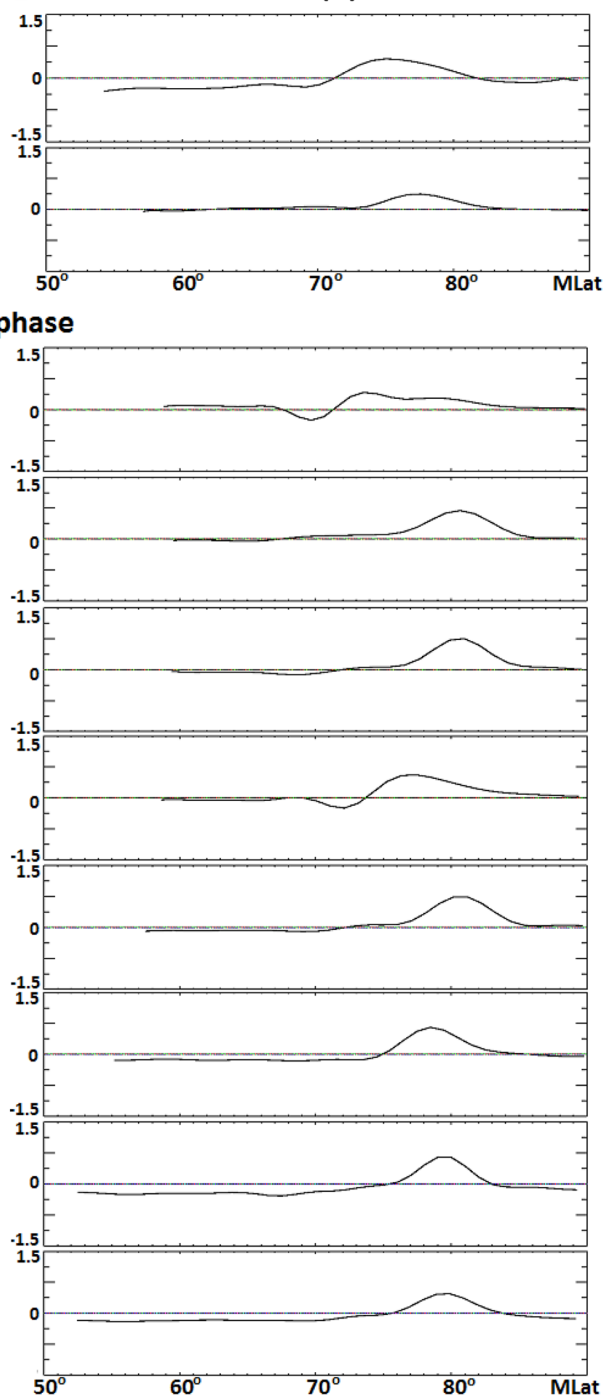

Figure A10. Direction and intensity values of the Hall current along the satellite orbit at the dawnside (a, 07:00-09:00 MLT, corresponding to the ascending section of the orbit) and duskside sectors (b, 19:00-21:00 MLT, descending orbit section). Positive currents denote an eastward current flow for the descending orbit section and a westward current for the ascending section, respectively. 
Author contributions. This paper is the result of longer, intense teamwork that we started several years ago after the CHAMP mission was completed. YIF had the principal idea for this kind of study and prepared the first manuscript draft (in Russian). PR provided the expertise and the software to deduce the electrojet current densities. LIG carried out most of the analysis and assembled all the figures. MF provided an English version of the manuscript and took care of the finalization of the study.

Competing interests. The authors declare that they have no conflict of interest.

Acknowledgements. The compilation of the storms and the corresponding IMF conditions during the intervals selected was conducted by use of the one-minute OMNI data base (http://omniweb.gsfc.nasa.gov/, last access: August 2018). The CHAMP mission was sponsored by the Space Agency of the German Aerospace Center (DLR) through funds of the Federal Ministry of Economics and Technology, following a decision of the German Federal Parliament (grant code 50EE0944). The data retrieval and operation of the CHAMP satellite by the German Space Operations Center (GSOC) of DLR are acknowledged.

The article processing charges for this open-access publication were covered by the Max Planck Society.

Edited by: Elias Roussos

Reviewed by: Jone Peter Reistad and one anonymous referee

\section{References}

Baumjohann, W. and Treumann, R. A.: Basic Space Plasma Physics, Imperial College Press, London, UK, 1996.

Chapman, S.: The electric current systems of magnetic storms, Terr. Magn. Atmos. Elect., 40, 349-370, 1935.

Chapman, S. and Bartels, J.: Geomagnetism, 1, Geomagnetic and related phenomena, Clarendon Press, Oxford, UK, 542 pp., 1940.

Cowley, S. W. H.: Dungey's Reconnection Model of the Earth's Magnetosphere: The first 40 Years, vol. 41 of Astrophysics and Space Science Proceedings, Springer International Publishing, Switzerland, 1-32, https://doi.org/10.1007/978-3-31918359-6_1, 2015.

Cowley, S. W. H. and Lockwood, M.: Excitation and decay of solar wind-driven flows in the magnetosphere-ionosphere system, Ann. Geophys., 10, 103-115, 1992.

Dungey, J. W.: Interplanetary magnetic field and the auroral zones, Phys. Rev. Lett., 6, 47-48, 1961.

Elphinstone, R. D., Murphree, J. S., and Cogger, L. L.: What is a global auroral substorm?, Rev. Geophys., 34, 169-232, 1996.

Feldstein, Y. I.: Magnetic field variations in the polar region during magnetically quiet periods and interplanetary magnetic fields, Space Sci. Rev., 18, 777-861, 1976.

Feldstein, Y. I. and Levitin, A. E.: Solar wind control of electric fields and currents in the ionosphere, J. Geomagn. Geoelectr., 38, 1143-1182, 1986.
Feldstein, Y. I., Lyatskaya, A. M., Sumaruk, P. V., and Shevnina, N. F.: Ionisation of the E-layer and the variations of the magnetic field in the near-polar region, Geomagn. Aeron.+, 15, 10211027, 1975a (in Russian).

Feldstein, Y. I., Sumaruk, P., and Shevnina, N. F.: To the diagnostics of the azimuthal component of the interplanetary magnetic field, C.R. Acad. Sci. URSS, 222, 833-836, 1975 b.

Feldstein, Y. I., Grafe, A., Gromova, L. I., and Popov, V. A.: Auroral electrojets during geomagnetic storms, J. Geophys. Res., 102, 14223-14235, https://doi.org/10.1029/97JA00577, 1997.

Feldstein, Y. I., Popov, V. A., Cumnock, J. A., Prigancova, A., Blomberg, L. G., Kozyra, J. U., Tsurutani, B. T., Gromova, L. I., and Levitin, A. E.: Auroral electrojets and boundaries of plasma domains in the magnetosphere during magnetically disturbed intervals, Ann. Geophys., 24, 2243-2276, https://doi.org/10.5194/angeo-24-2243-2006, 2006.

Friis-Christensen, E. and Wilhjelm, J.: Polar currents for different directions of the interplanetary magnetic field in the Y-Z plane, J. Geophys. Res., 80, 1248-1260, 1975.

Friis-Christensen, E., Lassen, K., Wilhjelm, J., Wilcox, J. M., Gonzales, W., and Colburn, D. S.: Critical Component of the Interplanetary Magnetic Field Responsible for Large Geomagnetic Effects in the Polar Cap, J. Geophys. Res., 77, 3371-3376, 1972.

Friis-Christensen, E., Kamide, Y., Richmond, A. D., and Matsushita, S.: Interplanetary magnetic field control of high-latitude electric fields and currents determined from Greenland magnetometer data, J. Geophys. Res., 90, 1325-1338, 1985.

Fukushima, N.: Generalized theorem for no ground magnetic effect of vertical currents connected with Pedersen currents in the uniform-conductivity ionosphere, Rep. Ionos. Space Res., 30, 35-40, 1976.

Galperin, Y. I. and Feldstein, Y. I.: Mapping of the precipitation regions to the plasma sheet, J. Geomagm. Geoelectr., 48, 857875, 1996.

Green, D. L., Waters, C. L., Korth, H., Anderson, B. J., Ridley, A. J., and Barnes, R. J.: Technique: Large-scale ionospheric conductance estimated from combined satellite and groundbased electromagnetic data, J. Geophys. Res., 112, A05303, https://doi.org/10.1029/2006JA012069, 2007.

Harang, L.: The Aurorae, The International Astrophysical Series edn., vol. 1, Chapman \& Hall, Ltd., London, UK, 163 pp., 1951.

Iijima, T. and Potemra, T. A.: The amplitude distribution of the field-aligned currents at northern high latitudes observed by TRIAD, J. Geophys. Res., 81, 2165-2174, 1976a.

Iijima, T. and Potemra, T. A.: Field-aligned currents in the dayside cusp observed by TRIAD, J. Geophys. Res., 81, 5971-5979, $1976 b$.

Iijima, T., Potemra, T. A., Zanetti, L. J., and Bythrow, P. F.: Large-scale Birkeland currents in the dayside polar region during strongly northward IMF: A new Birkeland current system, J. Geophys. Res., 89, 7441-7452, 1984.

Jørgensen, T. S., Friis-Christensen, E., and Wilhelm, J.: Interplanetary magnetic-field direction and high-latitude ionospheric currents, J. Geophys. Res., 77, 1976-1977, https://doi.org/10.1029/JA077i010p01976, 1972.

Kalegaev, V. V., Bakhmina, K. V., Alexeev, I. I., Belenkaya, E. S., Feldstein, Y. I., and Ganushkina, N. Y.: Asymmetry of the ring current during geomagnetic disturbances, Geomagn. Aeron.+, 48, 780-792, 2008 (in English). 
Kozyra, J. U. and Liemohn, M. W.: Ring current energy input and decay, Space Sci. Rev., 109, 105-131, 2003.

Laundal, K. M., Finlay, C. C., Olsen, N., and Reistad, J. P.: Solar Wind and Seasonal Influence on Ionospheric Currents From Swarm and CHAMP Measurements, J. Geophys. Res., 123, 4402-4429, https://doi.org/10.1029/2018JA025387, 2018.

Levitin, A. E., Afonina, R. G., Belov, B. A., and Feldstein, Y. I.: Geomagnetic variations and field-aligned currents at northern highlatitudes and their relations to solar wind parameters, Philos. T. Roy. Soc. A, 304, 253-301, 1982.

Mansurov, S. M.: New evidence of the relationship between magnetic field in space and on the Earth, Geomagn. Aeron.+, 9, 768773, 1969 (in English).

Milan, S. E.: Sun et Lumière: Solar wind-magnetosphere coupling as deduced from ionospheric flows and polar auroras, vol. 41 of Astrophysics and Space Science Proceedings,, Springer International Publishing, Switzerland, 33-64, https://doi.org/10.1007/978-3-319-18359-6_2, 2015.

Newell, P. T., Sotirelis, T., Liou, K., Meng, C.-I., and Rich, F. J.: A nearly universal solar wind-magnetosphere coupling function inferred from 10 magnetospheric state variables, J. Geophys. Res., 112, A01206, https://doi.org/10.1029/2006JA012015, 2007.

Olsen, N.: A new tool for determining ionospheric current from magnetic satellite data, Geophys. Res. Lett., 23, 3635-3638, 1996.

Reigber, C., Lühr, H., and Schwintzer, P.: CHAMP mission status, Adv. Space Res., 30, 129-134, 2002.

Richmond, A. D.: Ionospheric electrodynamics using magnetic Apex coordinates, J. Geomagn. Geoelectr., 47, 191-208, 1995.

Ritter, P., Lühr, H., Maus, S., and Viljanen, A.: High-latitude ionospheric currents during very quiet times: their characteristics and predictability, Ann. Geophys., 22, 2001-2014, https://doi.org/10.5194/angeo-22-2001-2004, 2004a.
Ritter, P., Lühr, H., Viljanen, A., Amm, O., Pulkkinen, A., and Sillanpää, I.: Ionospheric currents estimated simultaneously from CHAMP satelliteand IMAGE ground-based magnetic field measurements: a statisticalstudy at auroral latitudes, Ann. Geophys., 22, 417-430, https://doi.org/10.5194/angeo-22-417-2004, 2004b.

Ritter, P.: Hall current densities derived from CHAMP scalar magnetic field data during six magnetic storms 2003 and 2005, V. 1.0, GFZ Data Services, available at: http://doi.org/10.5880/GFZ.2.3. 2018.007, 2018.

Sandholt, P. E., Carlson, C., and Egeland, A.: Dayside and polar cap aurora, vol. 270 of Astrophys. Space Sci. Library, Kluwer Academ. Pub., Dordrecht/Boston/London, 287 pp., 2002.

Sandholt, P. E., Farrugia, C. J., and Denig, W. F.: Detailed dayside auroral morphology as a function of local time for southeast IMF orientation: implications for solar wind-magnetosphere coupling, Ann. Geophys., 22, 3537-3560, https://doi.org/10.5194/angeo22-3537-2004, 2004.

Sumaruk, P. V. and Feldstein, Y. I.: Sector structure of the interplanetary magnetic field and the magnetic variations in the near-polar region, Kosm. Issled., 11, 155-160, 1973 (in Russian).

Svalgaard, L.: Sector structure of the interplanetary magnetic field and daily variation of the geomagnetic field at high latitudes, in: Geophys. Papers, R-6, Danish Meteorol. Inst., Copenhagen, Denmark, 1968.

Wang, H., Lühr, H., Ridley, A., Ritter, P., and Yu, Y.: Storm time dynamics of auroral electrojets: CHAMP observation and the Space Weather Modeling Framework comparison, Ann. Geophys., 26, 555-570, https://doi.org/10.5194/angeo-26-555-2008, 2008.

Wilhjelm, J. and Friis-Christensen, E.: Electric field and high latitude zonal currents induced by merging of field lines, in: Geophys. Paper R-31, Dan. Meteorol. Inst., Charlottenlund, Denmark, 1971. 